\title{
Rapid "Turn-on" of Type-1 AGN in a Quiescent Early-type Galaxy SDSS1115+0544
}

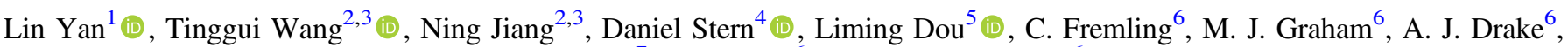 \\ Chenwei Yang ${ }^{7}$, K. Burdge ${ }^{6}$, and M. M. Kasliwal ${ }^{6}$ (D) \\ ${ }^{1}$ Caltech Optical Observatories, California Institute of Technology, Pasadena, CA 91125, USA; lyan@ @altech.edu \\ ${ }^{2}$ CAS Key Laboratory for Research in Galaxies and Cosmology, University of Science and Technology of China, Hefei, Anhui 230026, People's Republic of China \\ ${ }^{3}$ School of Astronomy and Space Science, University of Science and Technology of China, Hefei, Anhui 230026, People's Republic of China \\ ${ }^{4}$ Jet Propulsion Laboratory, California Institute of Technology, 4800 Oak Grove Drive, Mail Stop 169-221, Pasadena, CA 91109, USA \\ ${ }^{5}$ Center for Astrophysics, Guangzhou University, Guangzhou, 510006, People's Republic of China \\ ${ }^{6}$ Astronomy Department, California Institute of Technology, 1200 E. California Boulevard, CA 91225, USA \\ ${ }^{7}$ Polar Research Institute of China, 451 Jinqiao Road, Shanghai, 200136, People's Republic of China \\ Received 2018 November 27; revised 2019 February 5; accepted 2019 February 11; published 2019 March 20
}

\begin{abstract}
We present a detailed study of a transient in the center of SDSS1115+0544 based on the extensive UV, optical, mid-IR light curves (LCs) and spectra over 1200 days. The host galaxy is a quiescent early-type galaxy at $z=0.0899$ with a black hole mass of $2 \times 10^{7} M_{\odot}$. The transient underwent a 2.5 mag brightening over $\sim 120$ days, reaching a peak $V$-band luminosity (extinction corrected) of -20.9 mag, then fading 0.5 mag over 200 days, settling into a plateau of $>600$ days. Following the optical brightening are the significant mid-IR flares at 3.4 and $4.5 \mu \mathrm{m}$, with a peak time delay of $\sim 180$ days. The mid-IR LCs are explained as the echo of UV photons by a dust medium with a radius of $5 \times 10^{17} \mathrm{~cm}$, consistent with $E(B-V)$ of 0.58 inferred from the spectra. This event is very energetic with an extinction corrected $L_{\mathrm{bol}} \sim 4 \times 10^{44} \mathrm{erg} \mathrm{s}^{-1}$. Optical spectra over 400 days in the plateau phase revealed newly formed broad $\mathrm{H} \alpha, \beta$ emission with a FWHM of $\sim 3750 \mathrm{~km} \mathrm{~s}^{-1}$ and narrow coronal lines such as [Fe VII], [Ne V]. This flare also has a steeply rising UV continuum, detected by multi-epoch $S$ wift data at +700 to +900 days post optical peak. The broad Balmer lines and the UV continuum do not show significant temporal variations. The slow evolving LCs over 1200 days, the constant Balmer lines, and UV continuum at late times rule out tidal disruption event and SN IIn as the physical model for this event. We propose that this event is a "turn-on" AGN, transitioning from a quiescent state to a type-1 AGN with a sub-Eddington accretion rate of $0.017 M_{\odot} \mathrm{yr}^{-1}$. This change occurred on a very short timescale of $\sim 120-200$ days. The discovery of such a rapid "turn-on" AGN poses challenges to accretion disk theories and suggests more future detections of similar events.
\end{abstract}

Key words: galaxies: nuclei - stars: flare - supernovae: general

Supporting material: data behind figures

\section{Introduction}

In the past decade, time domain astronomy has made large strides forward with the advent of several wide area transient surveys, including the Panoramic Survey Telescope \& Rapid Response System (Kaiser et al. 2002), Palomar Transient Factory (PTF; Law et al. 2009; Rau et al. 2009), Catalina Realtime Transient Survey (CRTS; Drake et al. 2009), All-sky Automated Survey for Supernovae (ASASSN), ${ }^{8}$ Asteroid Terrestrial-impact Last Alert System (ATLAS; Tonry et al. 2018), and most recently, Zwicky Transient Facility (ZTF; Graham et al. 2019; Bellm et al. 2019). One particular area of these recent advances involves transients detected in the centers of galaxies, especially quiescent galaxies where no previous nuclear activities were detected. This type of transients includes both Tidal Disruption Events (TDEs) and Changing-look AGNs (CLAGNs). The first phenomenon involves the optical/UV flares generated by the disruption of a star when it gets too close to the central black hole and was theoretically predicted in 1980s (Hills 1976; Rees 1988; Phinney 1989). The second type, CLAGNs, is due to the change of AGN accretion rates. The changing states include transformation from quiescent, non-accreting black holes to active accreting type1 or type-2 AGNs (e.g., Gezari et al. 2017), or spectral change between a type-2 and a type-1 AGN (e.g., Ruan et al. 2016;

\footnotetext{
8 http://www.astronomy.ohio-state.edu/ assassin/index.shtml
}

Runnoe et al. 2016), or periodic photometric variations (several years timescale) in some AGNs (Oknyanskij 1978; Oknyanskij \& Lyuty 2007; Graham et al. 2015; Jun et al. 2015; Bon et al. 2016; Mathur et al. 2018). Both TDEs and CLAGNs have become the focus of many recent studies (van Velzen et al. 2011; Gezari et al. 2012, 2017; Yang et al. 2013, 2017; Arcavi et al. 2014; Holoien et al. 2014, 2016; MacLeod et al. 2016; Ruan et al. 2016; Runnoe et al. 2016; Blagorodnova et al. 2017a; Oknyansky et al. 2019). As the referee of this paper pointed out, spectral variations on timescales of years have been observed among nearby AGNs (e.g., NGC 4151, NGC 3516) since the 1960s (Andrillat 1968; Tohline \& Osterbrock 1976; Lyutyj et al. 1984; Penston \& Perez 1984). Possible detections of TDEs are found also among AGNs and infrared luminous galaxies (Kankare et al. 2017; Tadhunter et al. 2017; Mattila et al. 2018). The renewed interests may lead us to a deeper understanding of this subject since we are now supplemented with more statistics and better data from large transient surveys.

Photometric surveys cannot immediately distinguish these two types of transients without additional follow-up spectroscopy. This explains the recent surge of studies of CLAGNs; some are by-products from searches for TDEs. Observational separations of TDEs and CLAGNs are not always clear (e.g., Blanchard et al. 2017) due to the similar fundamental physics governing both types of sources. This confusion is also due to 
the small numbers of TDEs and CLAGNs discovered so far. The increasing sample sizes in the future should help us build more complete empirical characterizations and thus also a coherent picture of accretion physics explaining both types of flaring events.

After a half dozen of optical/UV TDEs were discovered, it was initially proposed that the host galaxies of TDEs are one specific type-post-starburst galaxies with characteristic spectral features of E+A galaxies (Arcavi et al. 2014; French et al. 2016). A hotly debated question is whether this is intrinsic or due to the TDE selection bias in the optical/UV wavelength. One way to address this question is to examine if using infrared variability could be an effective means to identify TDE candidates because it is less biased against dusty star-forming galaxies. This motivated us to carry out a systematic search for mid-infrared flare sources using the data taken by the Widefield Infrared Space Explorer (WISE; Wright et al. 2010) from 2009 to 2017. The detailed description of this search is prepared in a separate publication (N. Jiang et al. 2019, in preparation). Here we briefly summarize the sample. First, we impose four limits on our search. (1) We focus only on the Sloan Digital Sky Survey (SDSS) galaxies which have spectroscopic data. This gives us the critical information on the pre-flare properties of the host galaxies. (2) We limit our search to $z<0.2$. This condition is very conservative, and can be easily extended in future studies. (3) We only select sources whose mid-IR flares are still recent (i.e., their light curves [LCs] are either still rising or have just turned over the peak, but not yet reached completely quiescent levels). This criterion is chosen to give us sufficient time to take follow-up spectra. (4) We focus only on variations on timescales $>2$ weeks. Therefore, we can coadd the single frame photometries taken within one week time span, thus reducing the photometric errors. All single frame source photometry is made available by the WISE and NEOWISE-R (Mainzer et al. 2014) surveys through the IRSA data archive. ${ }^{9}$ We found a total of 28 galaxies with mid-IR flares with a magnitude change $>0.5 \mathrm{mag}$ (Vega). As shown in the histogram of WISE magnitude variation in Figure 1 of Stern et al. (2018), such a brightness change is significant, indicative of intrinsic physical variation. The sample LCs and the selection details are presented in Jiang et al. (2019, in preparation).

This paper is dedicated to a single object SDSS J111536.57 +054449.7 (hereafter SDSS1115+0544), which has a rich suite of data, including optical and mid-IR light curves, a spectral sequence over a timescale $>1 \mathrm{yr}$, and new Swift observations. As our analyses show, this object may represent a class of early-type galaxies, rapidly transforming from quiescent states over only a few hundred days to actively accreting type-1 AGNs. We demonstrate that there are some ambiguities between this type of transients and peculiar type II supernovae, and larger samples of such events over several years would help firmly establish the classification criteria.

\section{The Quiescent State of the Host Galaxy}

SDSS115+0544 has the sky position of R.A. = 11:15:36.57 and decl. $=05: 44: 49.73$ Equinox $\mathrm{J} 2000.0$ and at redshift of 0.08995. Before SDSS1115+0544 displayed optical and infrared flare in 2015, its quiescent state has multi-band

\footnotetext{
9 https://irsa.ipac.caltech.edu/cgi-bin/Gator/nph-scan?mission=irsa\&submit $=$ Select\&projshort $=$ WISE
}
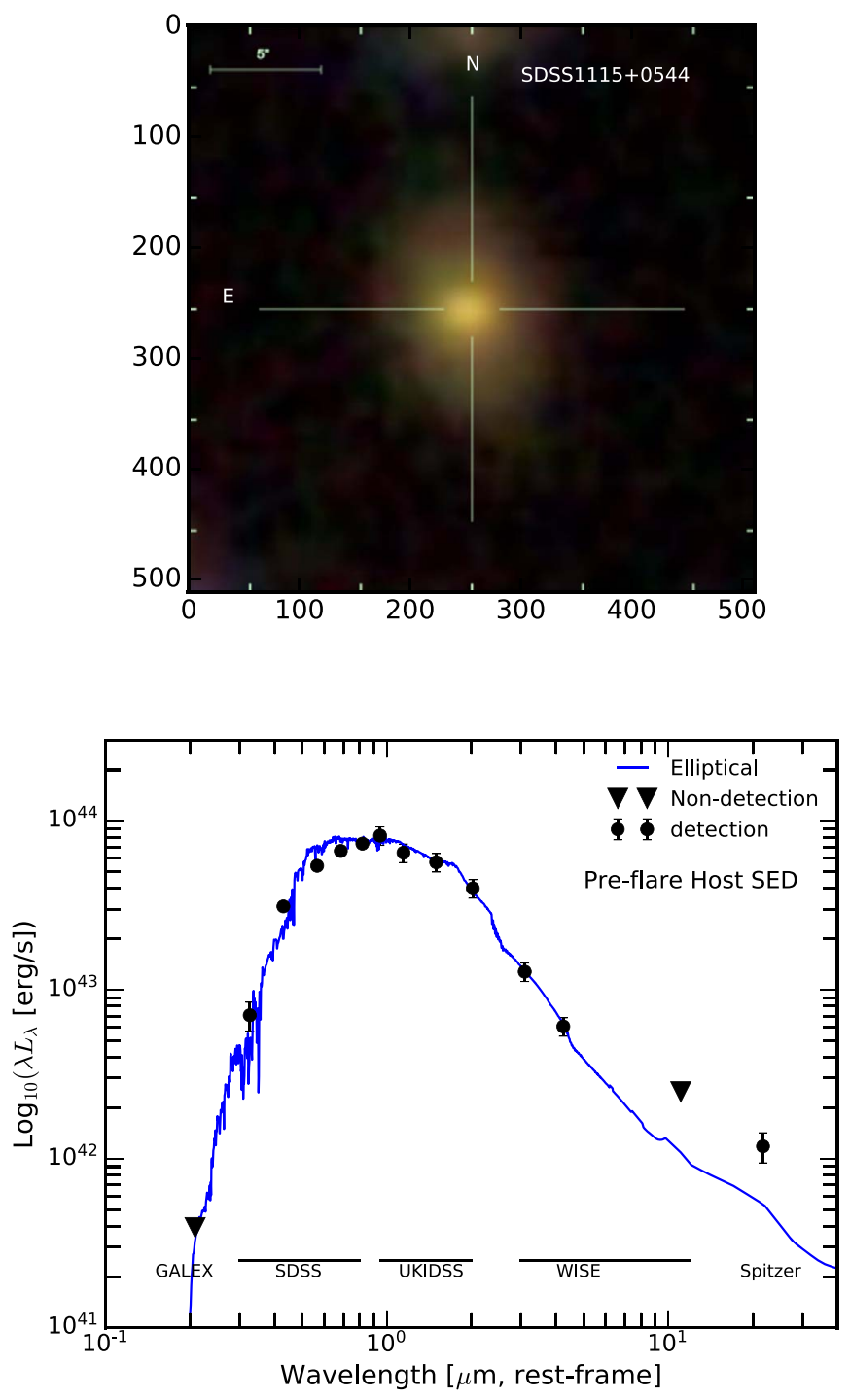

Figure 1. Two-panel plot shows the composite image, illustrating the morphological properties and the spectral energy distribution of the host galaxy SDSS1115+0544 in its quiescent state. The Spitzer $24 \mu \mathrm{m}$ flux is in excess of the quiescent early-type galaxy SED template, indicating dust obscured star formation, which is not accounted for by the model SED template.

photometry in ugriz (SDSS), YJHK (UKIDSS), and $3.4(W 1)$, $4.6(W 2), 12$ (W3, not detected), and $12 \mu \mathrm{m}$ (W4, not detected; ALLWISE), and $24 \mu \mathrm{m}$ (Spitzer). SDSS1115+0544 is a fairly bright, bulge-dominated galaxy with $g$ and $r$ magnitude of 17.96 and 17.06 mag (AB), respectively. Its pre-flare, panchromatic photometry is shown in the bottom panel of Figure 1, and at the optical/near-IR wavelength is well fit by the spectral energy distribution (SED) template of an elliptical galaxy (Polletta et al. 2007). In its quiescent state, the host galaxy was not detected in the near-UV (2267 $⿱$ ) ) by Galaxy Evolution Explorer (GALEX; >23.5 mag), in the WISE W3 and W4 bands, and in the FIRST $20 \mathrm{~cm}$ survey $(1 \sigma=0.15 \mathrm{mJy})$.

The pre-flare SDSS spectrum is shown in Figure 2, characteristic of a passive evolving early-type galaxy, with strong absorption lines such as $\mathrm{Ca} \mathrm{H}+\mathrm{K}, \mathrm{Ca} \mathrm{I}, \mathrm{Mg} \mathrm{I} 5175 \AA$ from a dominant old stellar population. The spectral models from the SDSS DR14 show that this galaxy has no current star formation activity. However, SDSS1115+0544 is detected by Spitzer at 


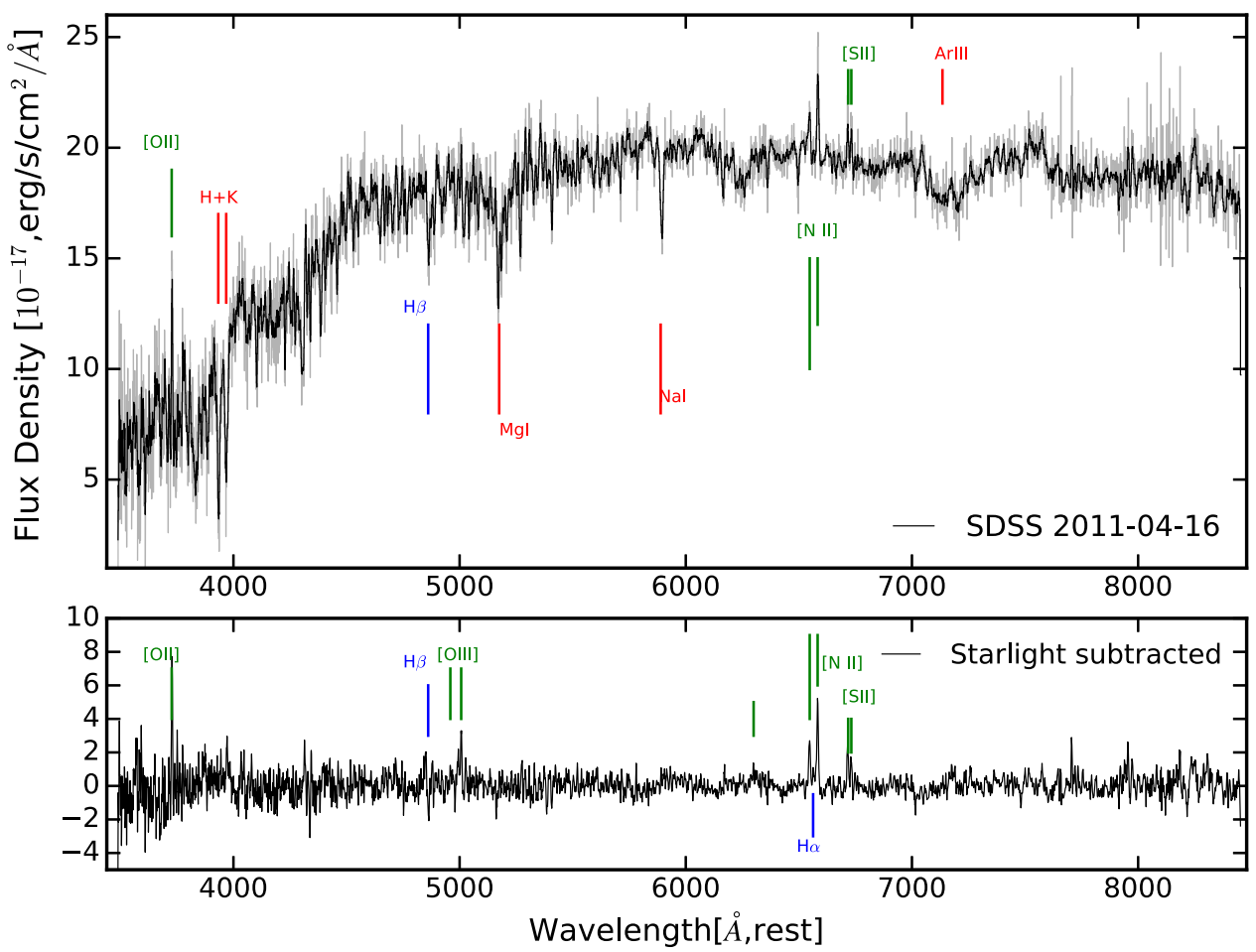

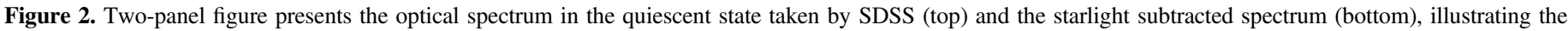
absorption lines and weak emission lines from the host galaxy. The line identifications are marked with vertical bars.

$24 \mu \mathrm{m}$ in 2006 and 2007. The dust obscured star formation rate (SFR) could be as much as $0.2 M_{\odot} \mathrm{yr}^{-1}$ based on the calibration relation $\operatorname{SFR}\left(M_{\odot} \mathrm{yr}^{-1}\right)=2.04 \times 10^{-43} \times \nu L_{\nu}\left(24 \mu \mathrm{m}, \mathrm{erg} \mathrm{s}^{-1}\right.$; Calzetti 2013). In comparison with the Milky Way SFR $\sim$ 0.68-1.45 $M_{\odot} \mathrm{yr}^{-1}$ (Robitaille \& Whitney 2010), SDSS1115 +0544 has a lower SFR.

The SDSS DR14 archive lists several derived parameters based on absorption lines-specifically, the velocity dispersion $\sigma_{v}$ is $128 \pm 17 \mathrm{~km} \mathrm{~s}^{-1}$ (Wisconsin method, Maraston models) and the stellar mass $M_{\text {star }}=3.47_{-0.52}^{+0.42} \times 10^{10} M_{\odot}$ is based on the Portsmouth method (passive model). Assuming the $\mathrm{M}-\sigma_{v}$ relation of $\log _{10}\left(M_{\mathrm{BH}}, \quad M_{\odot}\right)=8.32+5.64 \log _{10}(\sigma / 200)$ (McConnell \& Ma 2013), we infer the black hole mass of $\log _{10}\left(M_{\mathrm{BH}}\right)=7.29_{-0.11}^{+0.18}$.

Aside from absorption lines, SDSS115+0544 also displays weak nebular emission lines. To measure the reliable emission line ratios, we subtract the synthetic starlight spectrum, made by the FIREFLY models for the eBOSS spectra (SDSS DR14; Figure 2; Comparat et al. 2017). ${ }^{10}$ The starlight subtracted spectrum reveals [O III] 4959, $5007 \AA$, and [N II] 6548, $6583 \AA$ doublets, but very little $\mathrm{H} \beta$ and $\mathrm{H} \alpha$, yielding line ratios of $\log _{10}\left(\frac{[\mathrm{O} I I]}{\mathrm{H}_{\beta}}\right)>0.25$ and $\log _{10}\left(\frac{\mathrm{N} \text { II }}{\mathrm{H}_{\alpha}}\right)=0.23$. For comparison, star-forming galaxies generally have $\log _{10}\left(\frac{\mathrm{N}_{\text {II }}}{\mathrm{H}_{\alpha}}\right)<-0.2$, and strong AGNs have $\log _{10}\left(\frac{[\mathrm{O} \text { III] }}{\mathrm{H}_{\beta}}\right)>0.5$. SDSS1115+0544 falls within the lower-right region of the $[\mathrm{O} \mathrm{III}] / \mathrm{H}_{\beta}-\mathrm{N} \mathrm{II} / \mathrm{H}_{\alpha}$ diagram (i.e., the Baldwin, Phillips \& Telervich diagram). Kauffmann \& Heckman's (2005) classification of Low Ionization Nuclear Emission Line Regions (LINERs) have $\log _{10}\left(\frac{[\mathrm{O} \mathrm{III}]}{\mathrm{H} \beta}\right)<0.477$ and $\log _{10}\left(\frac{[\mathrm{N} \mathrm{II}]}{\mathrm{H} \alpha}\right)>-0.22$. The very

\footnotetext{
${ }^{10}$ https://www.sdss.org/dr14/spectro/eboss-firefly-value-added-catalog/
}

weak $\mathrm{H} \beta$ and $\mathrm{H} \alpha$ lines and the corresponding line ratios indicate that SDSS1115+0544 is aligned with the definition of a LINER, based on the diagnostics by Kewley et al. (2006) and Cid Fernandes et al. (2010). The SDSS DR14 archive also classified this object as a LINER.

The physical nature of the emission lines from a LINER is still a subject of debate. Some LINERs are thought to be weak AGNs. However, some emission lines in some LINERs are thought to be mainly produced by evolved stars during a short but very energetic post-AGB phase, as shown by a study of integral field spectroscopy of nearby LINERs (Singh et al. 2013). We will discuss the implications of the transient flare in a LINER in Section 6.

\section{Transient Observations in SDSS1115+0544}

\section{1. $L C s$}

SDSS1115+0544 has optical LC from CRTS (Drake et al. 2009), between 2006 January and 2017 May. The photometry comes from both the $1.5 \mathrm{~m}$ Mt. Lemmon Survey telescope and the $0.7 \mathrm{~m}$ Catalina Sky Survey telescope. We perform image subtraction analyses using the custom software, Fpipe (Fremling et al. 2016). The reference images are constructed using the CRTS images taken during the quiescent phase (before 2015). The transient photometry is measured by PSF fitting and calibrated onto the standard Johnson-Cousins $V$-band using SDSS photometry of the stars within the same images. Our optical photometry is in $\mathrm{AB}$ magnitude.

The mid-infrared photometry is from WISE (Wright et al. 2010) and NEOWISE (Mainzer et al. 2014) catalogs, covering a time span of 2010 to 2018. There is a gap between 2011 and 2013 because the WISE space telescope was placed in hibernation after the exhaustion of its cryogen at the end of the primary mission. Except this gap, the WISE has visited 

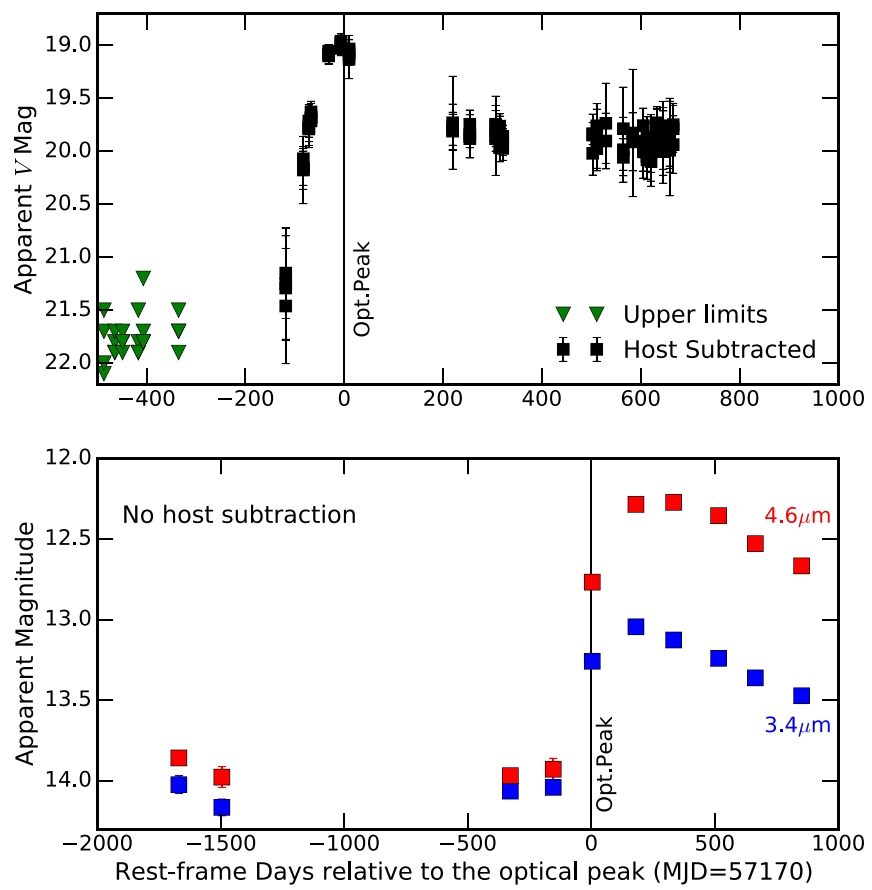

Figure 3. Observed (apparent magnitudes) light curves (LC) in the optical $V$-band (top) and the mid-infrared $3.4 \mu \mathrm{m}$ and $4.6 \mu \mathrm{m}$ (bottom). The optical LC is host subtracted (see details in the text), and the MIR LCs are the total apparent magnitudes. The data used to create this figure are available.

SDSSJ1115+0544 at $W 1(3.4 \mu \mathrm{m})$ and $W 2(4.6 \mu \mathrm{m})$ every half year, with 10-20 single exposures from each visit. We simply retrieved the $W 1$ and $W 2$ PSF profile-fit magnitudes from the catalogs, assuming that SDSS1115+0544 is a point-like source in the WISE images. We have searched the catalogs and there are no photometric contaminations from other sources within $10^{\prime \prime}$ of our target. Data with poor quality frame $\left(q i_{\text {fact }} \geqslant 0.5\right)$, charged particle hits $\left(s a a_{\text {sep }}<0\right)$, scattered moonlight $\left(\right.$ moon $\left._{\text {masked }}=1\right)$, and artifacts $\left(c c_{\text {flags }}>0\right)$ are rejected. The MIR photometry for SDSSJ1115+0544 is distributed over 10 epochs with intervals of $\sim 6$ months. No reliable short timescale variability is detected within each individual epoch, and thus we have binned the data every half year and computed the median values following our previous work (Jiang et al. 2016, 2017). The magnitudes in the first four epochs show little change. However, the fifth epoch presents a sudden brightening of $>0.5$ mag in both bands (Figure 3). The LCs reach their peak at the sixth epoch and then decline. The mean magnitudes of the first four epochs are $14.083 \pm 0.028$ and $13.91 \pm$ $0.041 \mathrm{mag}$ at $3.4 \mu \mathrm{m}$ and $4.6 \mu \mathrm{m}$, respectively. The [ $W 1-W 2]$ color in the quiescent state is $0.173,<0.8 \mathrm{mag}$, above which is the selection for mid-IR AGNs (Stern et al. 2012; Yan et al. 2013). This suggests that SDSS $1115+0544$ is a normal, nonAGN host in its quiescent state. We note here that mid-IR magnitudes are all in Vega system as provided by the WISE archive.

Figure 3 displays the optical (top) and mid-IR LCs (bottom) covering a time range of $\sim 12,000$ days. Here the apparent magnitudes are used to illustrate the brightness changes in the observed frame. The photometric flare in SDSS1115+0544 is significant and well captured by the CRTS and WISE observations.
Additional photometric data is from the Swift, including both UV Optical Telescope and X-ray Telescope (XRT). SDSS1115 +0544 is not detected in the UV by GALEX before the flare, and after the 2015 flare, it is significantly detected by Swift. The three epochs of Swift UV photometry were obtained on UT 2017 July 2, 2017 July 13, and 2017 December 26; see Table 1 for the details, which are at the tail end of optical and mid-IR flares. The data were reduced using the standard software tool, uvotimsum, provided by High Energy Astrophysics Software from NASA Goddard Space Flight Center. The photometry is measured using a $3^{\prime \prime}$ radius aperture, which is corrected out to $5^{\prime \prime}$ in radius for the total flux. The Swift XRT data were taken on the same three dates, and no detections were found at the position of SDSS1115+0544. Coadding all of the XRT data with a total of $4.27 \mathrm{ks}$, we derived a $1 \sigma$ upper limit of $7 \times 10^{-14} \mathrm{erg} \mathrm{s}^{-1} \mathrm{~cm}^{-2}$ in the Swift X-ray band.

\subsection{Spectroscopy}

The earliest spectrum is from SDSS taken in UT 2011 April 16. After discovering the strong mid-infrared flare in early 2017, we began our spectroscopic monitoring through UT 2018 May 13. The optical spectra were taken with both the Double Spectrograph (DBSP, Oke \& Gunn 1983) mounted on the Hale 200 inch telescope at Palomar Observatory as well as the Low Resolution Imager and Spectrograph (LRIS, Oke et al. 1995) on the Keck 10 meter telescope. These observations were taken under various different conditions; thus extra care is required when we compare line fluxes from different epochs as in Section 4.2.

The DBSP observations used the D550 dichroic, which splits the incoming light at $5500 \AA$ into the blue and red beam for two different grism/grating. The pixel scales are 0." 293 and 0". 398 per pixel for the red and blue CCD, respectively. The slit width of either $1^{\prime \prime}$ or 1 !" 5 was used depending on the seeing condition. All of the DBSP spectra were reduced using a python script written by Eric Bellm (University of Washington) and Kevin Burdge (Caltech). The spectra are flux calibrated using standard star observations from the same night. The Keck LRIS spectroscopy used the D560 dichroic in combination with the blue grism of 600 lines per mm, blazed at $4000 \AA$, and the red grating of 400 line per mm, blazed at $8500 \AA$. The long slit has a width of $1^{\prime \prime}$. This setup achieved a spectral resolution of $6.5-7.1 \AA$ for the full wavelength range of 3300-9500 $\AA$. The observation was taken under excellent weather, with seeing of 0 ". 6 and clear photometric conditions. The LRIS data was reduced by co-author Daniel Stern using his well-tested pipeline from previous studies. Table 2 shows the technical details of these observations.

Figure 4 compares the pre-flare (UT 2011 April 16 SDSS) and post-flare (UT 2018 May 13 LRIS) optical spectra, revealing striking newly formed features, including the rising UV continuum, prominent broad $\mathrm{H} \alpha$ emission lines, as well as high ionization metal emission lines. The post-flare Keck spectrum is available for download as an ascii table as the part of this paper. See full discussions in Section 4.2.

\section{Analyses and Results}

We convert the temporal variations in apparent brightness (Figure 3 ) to host-subtracted absolute magnitudes and monochromatic luminosity $\lambda L_{\lambda}$. The results are shown in Figure 5. For both optical and mid-IR, the host-subtracted LCs are 
Table 1

The Swift UV Photometry

\begin{tabular}{|c|c|c|c|c|c|c|c|}
\hline Obs.Date & $\begin{array}{l}\text { MJD } \\
\text { (days) }\end{array}$ & Filter & $\begin{array}{c}\nu \\
\left(10^{15} \mathrm{~Hz}\right)\end{array}$ & $\begin{array}{c}f_{\nu} \\
(\mathrm{mJy})\end{array}$ & $\begin{array}{c}\delta f_{\nu} \\
(\mathrm{mJy})\end{array}$ & $\begin{array}{c}\text { AB mag } \\
(\mathrm{mag})\end{array}$ & $\begin{array}{l}\delta \mathrm{mag} \\
(\mathrm{mag})\end{array}$ \\
\hline 2017 Jul 2 & 57936.943 & UVW2 & 1.475 & 0.0084 & 0.0016 & 21.59 & 0.21 \\
\hline 2017 Jul 2 & 57936.933 & UVM2 & 1.345 & 0.0141 & 0.0023 & 21.03 & 0.18 \\
\hline 2017 Jul 2 & 57936.953 & UVW1 & 1.157 & 0.0209 & 0.0029 & 20.58 & 0.15 \\
\hline 2017 Jul 13 & 57947.872 & UVW2 & 1.475 & 0.0124 & 0.0017 & 21.16 & 0.15 \\
\hline 2017 Jul 13 & 57947.875 & UVM2 & 1.345 & 0.0114 & 0.0019 & 21.26 & 0.18 \\
\hline 2017 Jul 13 & 57947.878 & UVW1 & 1.157 & 0.0207 & 0.0026 & 20.60 & 0.14 \\
\hline 2017 Dec 26 & 58112.816 & UVW2 & 1.475 & 0.0120 & 0.0019 & 21.20 & 0.17 \\
\hline 2017 Dec 26 & 58112.817 & UVM2 & 1.345 & 0.0121 & 0.0022 & 21.19 & 0.20 \\
\hline 2017 Dec 26 & 58112.819 & UVW1 & 1.157 & 0.0174 & 0.0030 & 20.79 & 0.18 \\
\hline
\end{tabular}

Table 2

Spectroscopic Observation Log

\begin{tabular}{lccccc}
\hline \hline Obs. Date & $\begin{array}{c}\text { MJD } \\
\text { (days) }\end{array}$ & $\begin{array}{c}\text { Phase }^{\mathrm{a}} \\
(\text { days })\end{array}$ & Instrument & $\begin{array}{c}\Delta \lambda \\
(\AA)\end{array}$ & Inst. Res. $^{\mathrm{o}^{\mathrm{O}}}$ \\
\hline 2011 Apr 16 & 52326 & -4444 & SDSS & $3100-9000$ & 1800 \\
2016 Jan 6 & 57393 & 204.6 & LAMOST & $3100-9000$ & 2500 \\
2017 May 24 & 57898 & 668.0 & DBSP & $3100-9500$ & $700 / 1200$ \\
2017 Nov 11 & 58068 & 823.9 & DBSP & $3100-9500$ & $700 / 1200$ \\
2017 Nov 25 & 58081 & 835.9 & DBSP & $3400-9500$ & $700 / 1200$ \\
2018 Jan 14 & 58132 & 882.7 & DBSP & $3400-9500$ & $700 / 1200$ \\
2018 Apr 11 & 58218 & 961.6 & DBSP & $3100-9500$ & $700 / 1200$ \\
2018 May 13 & 58251 & 992.3 & LRIS & $3100-10000$ & $1000 / 1000$ \\
\hline
\end{tabular}

Notes.

a The rest-frame phases is relative to the peak dates of MJD $=57170$.

${ }^{\mathrm{b}}$ Instrument resolution is shown for blue and red side, computed as $\lambda / \delta \lambda$ at 4000 and $7000 \AA$

available for download in ascii tables. The apparent magnitudes as functions of time are included with the explanatory information in the headers. The absolute magnitudes are calculated from $\mathrm{m}-\mathrm{M}=5 \log _{10}\left(D_{\mathrm{L}} / 10 \mathrm{pc}\right)+\mathrm{KC}$, where $D_{\mathrm{L}}=414 \mathrm{Mpc}$ is the co-moving luminosity distance and $\mathrm{KC}$ is the $\mathrm{K}$-correction. At $z=0.0895, \mathrm{KC}$ is relatively small ( $\sim 0.1$ mag; e.g., Hogg et al. 2002) and ignored by our analysis, as our mid-IR flares are not measured from image subtractions; thus the systematic uncertainties in the MIR LCs could be as large as $0.05 \mathrm{mag}$. Mid-IR Vega magnitudes are converted to the $\mathrm{AB}$ system to be consistent with that of optical LC. The transformation between $[W 1, W 2]($ vega $)-[W 1, W 2]$ $(\mathrm{AB})=[-2.65,-3.29] .^{11}$ The conversion factor in $W 2$ is large, which explains why the flare has smaller $W 1-W 2$ color in $\mathrm{AB}$ magnitude than in the Vega system.

In addition, the apparent magnitudes are converted into flux densities $f_{\lambda}$ in $\mathrm{erg} \mathrm{s}^{-1} \mathrm{~cm}^{-2} \AA^{-1}$ using the zero points tabulated in the WISE Explanatory Supplement. The mid-IR photometry at the first four epochs does not vary, and the galaxy is in the quiescent state (Figure 3). The mean magnitudes of these four epochs are taken to represent the host galaxy emission at the level of $\langle W 1\rangle=14.075$ and $\langle W 2\rangle=13.934 \mathrm{mag}$, respectively. These host emissions are removed to produce the hostsubtracted LCs. The three panels in Figure 5 illustrate several important results, which are discussed in the following subsections.

\footnotetext{
11 http://wise2.ipac.caltech.edu/docs/release/allsky/expsup/
}

\subsection{Mid-infrared Time Lag}

The mid-infrared flare is clearly delayed by about $\sim 180$ days ( $\left.\Delta t_{\text {delay }}\right)$ relative to the optical flare (peak-to-peak). Independent of the physical processes of the central UV/optical flare, the midinfrared LCs are likely the product of the dust echo of the UV/ optical flares. The basic idea is that UV/optical photons travel a distance $R$ and get absorbed by the dust grains, which in turn reradiate the excess energy in the infrared. Roughly speaking, the time delay $\Delta t_{\text {delay }}$ is $\sim R / c$, where $c$ is the speed of light. $\Delta t_{\text {delay }} \sim 180$ days implies that the dusty medium is at a distance of $\sim 5 \times 10^{17} \mathrm{~cm}$ from the UV/optical source. For comparison, the dust sublimation radius is $R_{\mathrm{sub}}(p c)=1.3\left(L_{\mathrm{UV}} / 10^{46} \mathrm{erg} \mathrm{s}^{-1}\right)^{0.5}$ $\left(T_{\mathrm{sub}} / 1500 \mathrm{~K}\right)^{-2.8}(a / 0.05 \mu \mathrm{m})^{-0.5}$, with $a$ the size of dust grain and $T_{\text {sub }}$ the grain sublimation temperature (Barvainis 1987). With the extinction corrected $L_{\mathrm{UV}} \sim 1 \times 10^{44} \mathrm{erg} \mathrm{s}^{-1}$ (see Section 4.3), and assuming graphite dust grains (their $T_{\text {sub }}=1500 \mathrm{~K}$ and $a=0.05 \mu \mathrm{m})$, we have the sublimation radius $R_{\text {sub }}=0.13 \mathrm{pc}=4 \times 10^{17} \mathrm{~cm}$, which is comparable to the radius of the dust shell computed from the time lag between the optical and mid-IR LCs.

The optical and mid-IR LCs also constrain the geometry, orientation, and thickness of the dusty gas. For example, the fact that optical and UV flare signals are observed can rule out the spherical dust distribution with high filling factors (or high optical depths). Detailed modelings of the LC shape can constrain the physical parameters of the dusty medium. One simple example is the tilted dust ring that is inferred from the mid-IR flare in IRAS F01004-2237 (Dou et al. 2017). For our sample of mid-IR flares, the LC modeling is planned for a separate paper (T. Wang et al. 2019, in preparation).

\subsection{Dramatic Spectral Changes}

A rich set of new spectral features emerged in the post-flare optical spectra (Figure 4). This includes strong and broad $\mathrm{H} \alpha$, $\mathrm{H} \beta$, and He I $5875 \AA$; steeply rising UV continuum $(\lambda>3500 \AA)$; narrow metal lines; and most interestingly, [Fe VII] coronal lines. Inspection of the two-dimensional spectra found that $\mathrm{H} \alpha$ is from the central spatially unresolved region. This rules out supernova exploded at a distance $>1.5^{\prime \prime}$ from the center (angular size distance of $2.5 \mathrm{kpc}$ ).

Six optical spectra were obtained between UT 2017 May 24 and 2018 May 13 (Table 2). The P200 spectra were extracted using an aperture of $4^{\prime \prime}$, roughly 2.5-3 times the typical seeing. This is large enough to capture most of the line emission from the central point source. For comparison, the Petrosian radius 


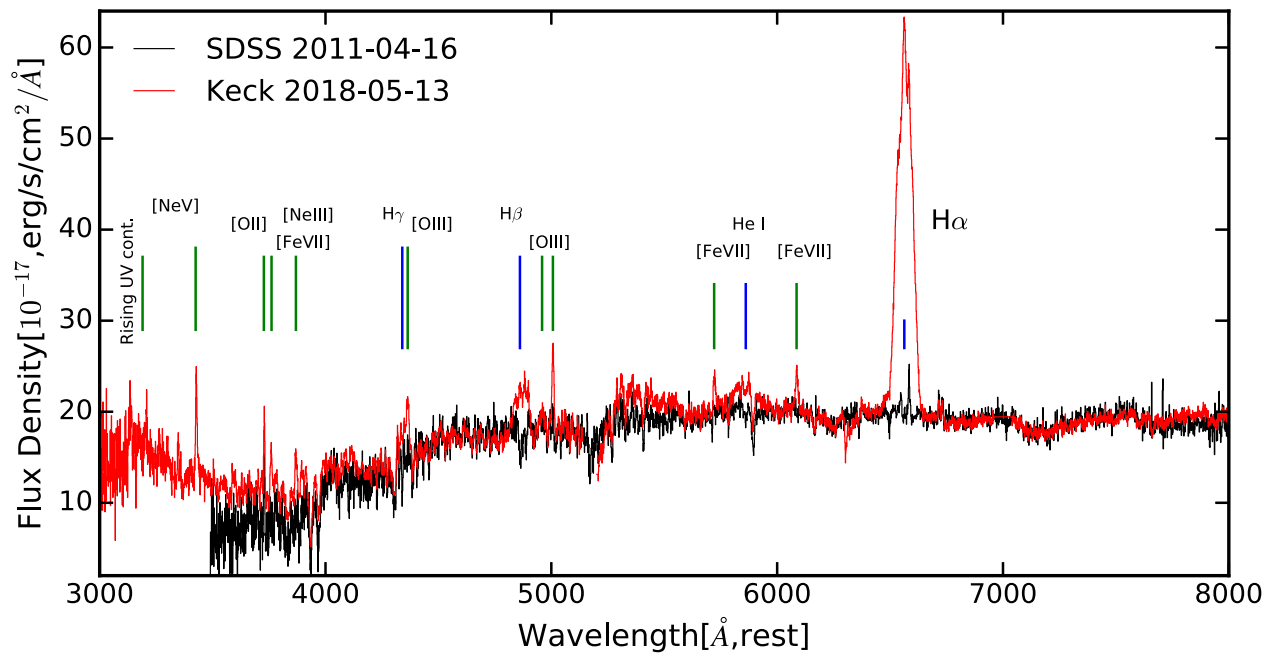

Figure 4. Observed Keck spectrum on UT 2018 May 13 (red) in comparison with the quiescent state SDSS spectrum from UT 2011 April 16 (black). The prominent rising UV continuum and new emission lines are marked. We note that the Keck spectrum shown here is made available for download as an ascii table in this paper. The data used to create this figure are available.

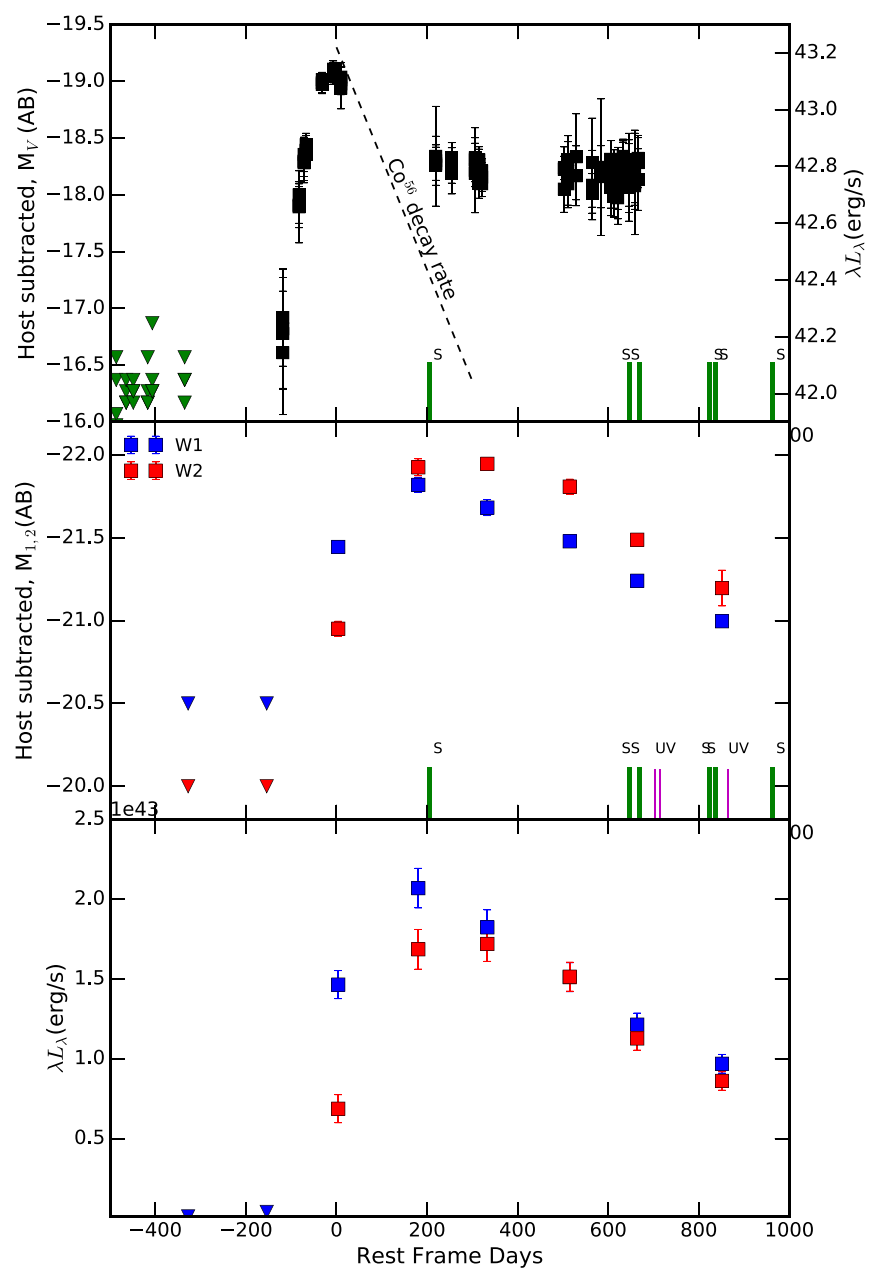

Figure 5. Optical and mid-infrared light curves of SDSS1115+0544 presented in host-subtracted absolute magnitudes $(\mathrm{AB})$ and luminosities over a time span of $\sim 1200$ days. The downward triangle symbols are the non-detection limits. The vertical green bar with a letter "S" marks the epoch with the spectroscopic data. In addition, the vertical purple bar marks the time when Swift UV photometry was taken. Dashed lines mark the fading slope of radioactive decay rate from $\mathrm{Co}^{56}$. containing $50 \%$ of the total flux is $1 . " 8$ for the host galaxy SDSS1115+0544. Each flux calibrated spectrum is matched to the SDSS spectral continuum and $\mathrm{Ca} \mathrm{H}+\mathrm{K}$ absorption features to produce the host galaxy subtracted, flare spectrum. Figure 6 displays the host-subtracted spectra from four different epochs. Figure 7 shows the $\mathrm{H} \alpha$ profiles observed in multi-epochs. Broadly speaking, these flare spectra do not show prominent variation during a 1-year period from 2017 to 2018. The last epochal spectrum was taken with LRIS on the Keck under extremely good conditions, with a clear sky and 0.6 seeing. The Keck spectrum was extracted with a 1 !! $5(2.5 \times$ seeing $)$ aperture, capturing most of the $\mathrm{H} \alpha$ emission from the central unresolved region, with the total line flux of $2.1 \times$ $10^{-14} \mathrm{erg} \mathrm{s}^{-1} \mathrm{~cm}^{-2}$; thus $L_{\mathrm{H} \alpha}=4 \times 10^{41} \mathrm{erg} \mathrm{s}^{-1}$.

All emission-line measurements are made with the hostsubtracted spectra. Table 3 tabulates the line wavelengths, integrated fluxes, and line widths (FWHM) measured from the Keck spectrum (UT 2018 May 13).

\subsubsection{High Dust Content and the Inferred $\mathrm{E}(\mathrm{B}-\mathrm{V})$}

In its quiescent state, SDSS1115+0544 was detected by Spitzer at $24 \mu \mathrm{m}$, with the flux in access to the SED of a pure early-type galaxy, as shown in Figure 1. This mid-infrared excess suggests that the galaxy contains a substantial amount of warm dust.

The UV/optical flare ionized neutral hydrogen, producing Balmer recombination lines. In the Keck spectrum, the $\mathrm{H} \alpha$-to$\mathrm{H} \beta$ line ratio is 5.45 , significantly larger than 3.1 , a value commonly found for blue AGNs (Dong et al. 2008). We adopt the relation derived by Caplan \& Deharveng (1986), $A_{\mathrm{H} \alpha}=$ $5.25\left[\log _{10}(\mathrm{H} \alpha / \mathrm{H} \beta)-\log _{10}\left(2.86 t_{e}^{-0.07}\right)\right]$, where $\mathrm{H} \alpha / \mathrm{H} \beta$ is the line flux ratio and $t_{e}$ is the electron temperature in units of $10^{4} \mathrm{~K}$. The standard extinction curve with $R_{V}=A_{V} /$ $E(B-V)=3.1$ (Table 7.1 Osterbrock \& Ferland 2006) gives $A_{\mathrm{H} \alpha} / A_{V}=0.82$. Combining these two equations, we derive $E(B-V)=0.58$ and $A_{V}=1.8 \mathrm{mag}$. The extinction corrected $V$-band peak luminosity is $\nu L_{\nu}(V)=7 \times 10^{43} \mathrm{erg} \mathrm{s}^{-1}$, making it comparable to that of most luminous supernovae and TDEs.

Dust absorption can also explain the Swift X-ray non-detections. The flux limit is $7 \times 10^{-14} \mathrm{erg} \mathrm{s}^{-1} \mathrm{~cm}^{-2}$ at the $90 \%$ confidence, 


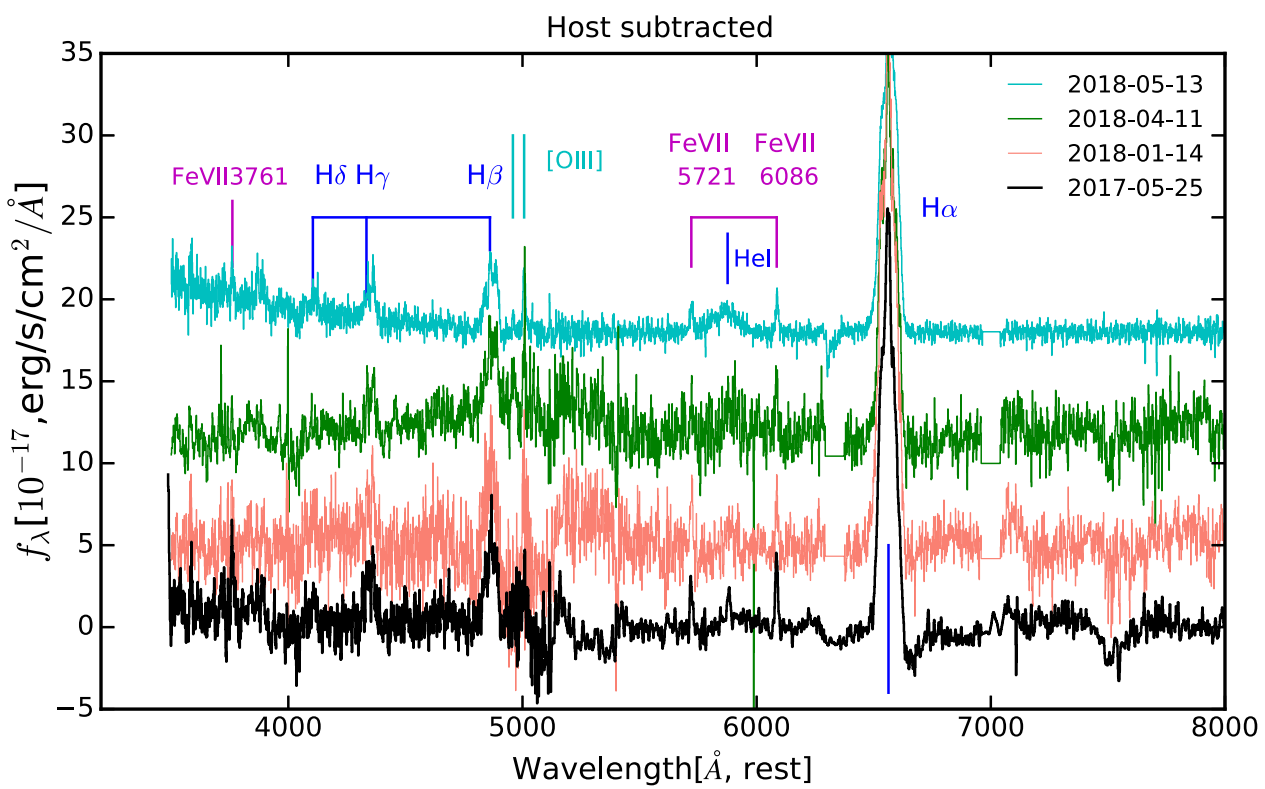

Figure 6. Host galaxy subtracted spectra are displayed. The data are from four epochs in 2017-2018, after the peak of the optical flare. All prominent new spectral emission lines are marked with vertical bars.

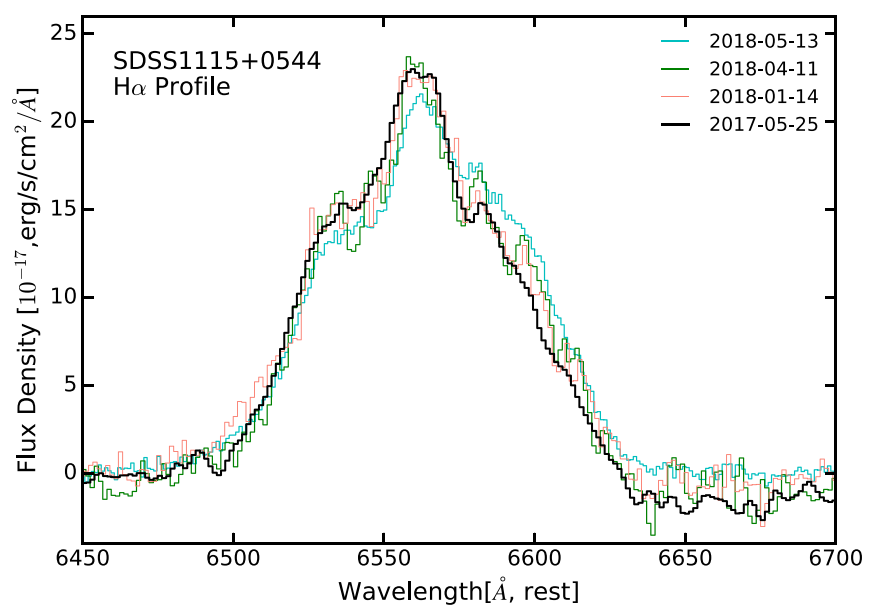

Figure 7. Zoom-in display of the $\mathrm{H} \alpha$ spectral region from the $\mathrm{P} 200$ spectra at four epochs spanning 1 year.

corresponding to the luminosity limit of $1.4 \times 10^{42} \mathrm{erg} \mathrm{s}^{-1}$. A previous study of a sample of 63 AGNs (Gelbord et al. 2009) has found that the soft X-ray luminosity is correlated with the coronal line luminosity, with $\log _{10}\left[f_{\text {x-ray }} / f([\mathrm{Fe} V \mathrm{VII}])\right]=(3.37 \pm 0.51)$. This sets the expected intrinsic soft $\mathrm{X}$-ray luminosity to $(0.5-5.3) \times 10^{43} \mathrm{erg} \mathrm{s}^{-1}$, which is higher than the Swift nondetection limit by a factor of (3-30). Non-detection in X-ray can be explained if the expected intrinsic soft X-ray flux is absorbed by a gas column density of $3 \times 10^{21} \mathrm{~cm}^{-2}$.

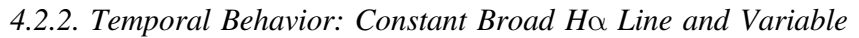 Narrow [O III] 5007 and $[\mathrm{Fe}$ VII] $6086 \AA$ lines}

We measured the broad $\mathrm{H} \alpha$ line fluxes using the four epochal P200 spectra and do not find significant temporal variations, with the line fluxes in a narrow range, $(1.9,2.7,1.8$, $2.0) \times 10^{-14} \mathrm{erg} \mathrm{s}^{-1}$ for UT 2018 April 11, 2018 January 14, 2017 November 24, and 2017 May 24, respectively. These values are similar to the integrated flux measured from the Keck spectrum as well. In addition, SDSS1115+0544 was
Table 3

Emission Line Measurements ${ }^{\mathrm{a}}$

\begin{tabular}{|c|c|c|c|}
\hline Line Name & Wavelength & $\begin{array}{l}\text { Line Flux } \\
\mathrm{erg} \mathrm{s}^{-1} \mathrm{~cm}^{-2}\end{array}$ & $\begin{array}{c}\text { FWHM } \\
\AA\end{array}$ \\
\hline $\mathrm{H} \alpha$ & 6563 & $(1.7 \pm 0.007) \mathrm{e}-14$ & 85 \\
\hline $\mathrm{H} \beta$ & 4861 & $(2.8 \pm 0.06) \mathrm{e}-15$ & 78 \\
\hline $\mathrm{H} \gamma$ & 4341 & $(5.9 \pm 0.6) \mathrm{e}-16$ & 53 \\
\hline He I & 5876 & $(1.9 \pm 0.07) \mathrm{e}-15$ & 60 \\
\hline$[\mathrm{Fe} \mathrm{X}]^{\mathrm{a}}$ & 6373 & $<9.2 \mathrm{e}-17$ & $<7.9$ \\
\hline [Fe VII $]$ & 6087 & $(3.6 \pm 0.3) \mathrm{e}-16$ & 16.9 \\
\hline [Fe VII] & 5721 & $(3.1 \pm 0.3) \mathrm{e}-16$ & 15.2 \\
\hline [O III] & 5007 & $(6.2 \pm 0.1) \mathrm{e}-16$ & 12.0 \\
\hline [O III] & 4959 & $(1.9 \pm 0.1) \mathrm{e}-16$ & 14.0 \\
\hline [O III] & 4364 & $(3.3 \pm 0.2) \mathrm{e}-16$ & 11.5 \\
\hline [Ne III] & 3869 & $(2.7 \pm 0.2) \mathrm{e}-16$ & 11.4 \\
\hline [Fe VII $]$ & 3760 & $(2.4 \pm 0.3) \mathrm{e}-16$ & 10.3 \\
\hline$[\mathrm{Ne} \mathrm{V}]^{b}$ & 3425 & $(7.6 \pm 0.4) \mathrm{e}-16$ & 9.0 \\
\hline$[\mathrm{Ne} \mathrm{V}]^{b}$ & 3346 & $(2.8 \pm 0.3) \mathrm{e}-16$ & 7.2 \\
\hline $\mathrm{He}$ II $^{b}$ & 3203 & $(2.3 \pm 0.3) \mathrm{e}-16$ & 7.3 \\
\hline $\mathrm{O} \mathrm{III}^{b}$ & 3133 & $(4.4 \pm 0.5) \mathrm{e}-16$ & 11.9 \\
\hline [S II] & 6731 & $5.5 e-17$ & 6.3 \\
\hline [S II $]$ & 6717 & $9.4 \mathrm{e}-17$ & 9.8 \\
\hline [O II] & 3727 & $4.0 \mathrm{e}-16$ & 5.5 \\
\hline
\end{tabular}

Note.

a All of the line measurements are done using the Keck spectrum taken on 2018 May 13.

observed by LAMOST (spectral fiber diameter 3") on UT 2016 January 6 (Yang et al. 2017). With the same method, we measured the $\mathrm{H} \alpha$ line flux of $2.0 \times 10^{-14} \mathrm{erg} \mathrm{s}^{-1}$. We infer $L_{\mathrm{H} \alpha} \sim 4 \times 10^{41} \mathrm{erg} \mathrm{s}^{-1}$. The broad H $\alpha$ FWM has a velocity width of $\sim 3750 \mathrm{~km} \mathrm{~s}^{-1}$ after corrected for the instrumental resolution. Similar to the constant line flux, the line width does not change with time. $\mathrm{He} \mathrm{I} 5876 \AA$ and $\mathrm{H} \beta$ line widths are consistent with that of $\mathrm{H} \alpha$.

Figure 8 compares the local continuum subtracted spectra around [O III] $5007 \AA$ doublet and [Fe VII] 5721, $6082 \AA$ lines pre- and post-flare. It is clear that after the flare, [O III] $5007 \AA$ 

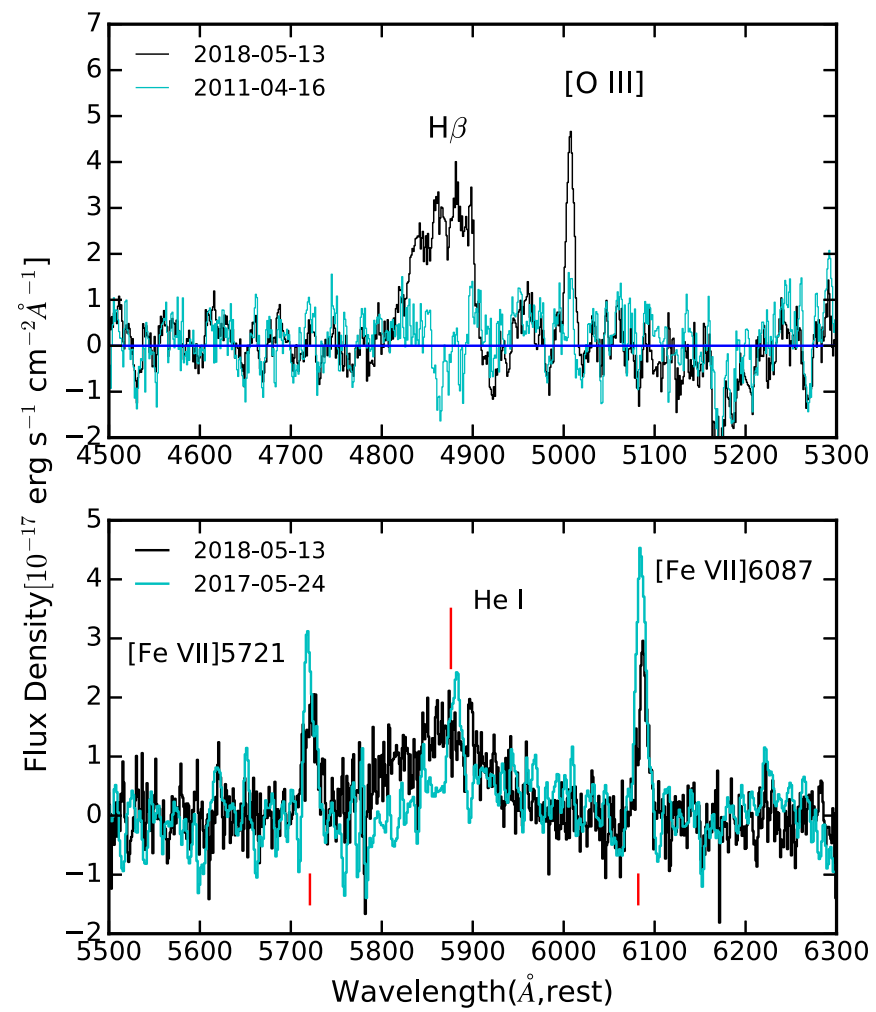

Figure 8. Narrow metal lines, [O III] $5007 \AA$ and coronal [Fe VII], are displayed at the pre-flare (UT 2011 April 16, cyan) and the post-flare (UT 2018 May 13, black) phase. This comparison directly illustrates the temporal change.

became stronger, with line flux roughly a factor of two higher than that of the SDSS spectrum from 2011. For DBSP spectra, [O III] $5007 \AA$ falls near the D550 dichroic beam splitter; thus no reliable information is available. We interpret the line flux increase between these two epochs is due to the increase of the ionization continuum. [Fe VII] $57216087 \AA$ lines are detected at four different epochs. Using the same method, we measured $f_{[\mathrm{Fe} \text { VII] } 6087}=(5.8,4.3,4.1,3.8) \times 10^{-16} \mathrm{erg} \mathrm{s}^{-1} \mathrm{~cm}^{-2}$ at UT 2017 May 24, 2018 January 14, 2018 April 11, and 2018 May 13, respectively. We conclude that coronal line flux is decreasing with time. The [Fe VII] line flux changes in these four epochs echo the variation of the ionization continuum with time (optical LC) and show time delays in relation to the continuum changes because ionizing photons need time to travel the size of the narrow line regions. For details, see Section 4.2.3. As noted in many studies of local AGNs displaying temporal spectral variabilities (e.g., NGC 548 and NGC 4151; Landt et al. 2015; Rose et al. 2015; Oknyansky et al. 2019), [Fe X] variability is usually associated with that of [Fe VII]. However, [Fe X] line is not detected in our data. Section 5.3 includes detailed discussions on the various coronal lines.

\subsubsection{Sizes, Gas Density, and Temperature of the Broad and Narrow Line Emitting Regions (NLRs)}

Regardless of what physical processes are producing the flare, several properties of the broad and NLRs can be inferred from time lags between continuum and spectral lines and line ratio diagnostics.
The energetic UV/optical flare is likely to drive kinematic motions. The broad Balmer emission lines probably come from photoionized gas clumps moving at a velocity of $3000-4000 \mathrm{~km} \mathrm{~s}^{-1}$. We can estimate the size of broad line region (BLR) using $\Delta t \times v_{\text {outflow }}$; here $\Delta t$ is time and $v_{\text {outflow }}$ is cloud velocity. If we take $\Delta t \leqslant 300$ days, the time between the rise time of the optical flare and the first detection of $\mathrm{H} \alpha$ (LAMOST data), and assuming clouds moving at $4000 \mathrm{~km} \mathrm{~s}^{-1}$, we have a BLR radius of $1 \times 10^{16} \mathrm{~cm} \sim 0.003 \mathrm{pc}$.

The size of the NLR can be estimated in two ways, using time lag and kinematics. The [O III] and [Fe VII] temporal behaviors can be understood in the context of the time variation of the ionizing continuum. The post-flare [O III] $5007 \AA$ line flux is higher than pre-flare because more ionized $\mathrm{O}^{+4}$ ions are produced by the UV/optical flare photons. For a NLR with a radius of $R_{N}$, the UV ionizing photons will take $\Delta t=R_{N} / c$ to travel from the center to the line emitting ions. Therefore, there is a time delay of $\Delta t$ between the productions of UV ionizing photons and narrow emission lines. The [Fe VII] line fluxes are stronger at UT 2017 May 24 than UT 2018 May 13. This is due to higher ionizing UV continuum at the LC peak, between 2015 May and 2016 May, prior to the $V$-band LC plateau. The UV ionizing continuum responsible for the 2018 May 13 [Fe VII] emission is likely from the time when the LC has faded, reaching to the plateau in 2017. Our data can only give an approximate limit on $\Delta t<770$ days. The narrow region has a size $R_{N}<2 \times 10^{18} \mathrm{~cm} \sim 0.6 \mathrm{pc}$.

Another way to estimate the NLR size is to use the measured [Fe VII] line width. From the Keck spectrum on 2018 May 13, the [Fe VII] $6087 \AA$ FWHM is $\sim 15.5 \AA$ (rest-frame), which translates to a velocity of $\sim 770 \mathrm{~km} \mathrm{~s}^{-1}$. Taking into account of the LRIS-R spectral resolution $\Delta \lambda=6.9 \AA$, we have the intrinsic line velocity width $v=703 \mathrm{~km} \mathrm{~s}^{-1}$. Assuming this line width is due to narrow line emitting gas moving in the gravitational potential of a $2 \times 10^{7} M_{\odot}$ black hole, we have $R=\frac{M G}{v^{2}}=5 \times 10^{17} \mathrm{~cm}$. These two crude estimates are roughly consistent, and the NLR is more than an order of magnitude larger than the BLR.

Regardless of the physical nature of the flare, the observed line ratios from [Fe VII] and [O III] can be used to constrain the gas density and temperature, as described in standard text books (e.g Osterbrock \& Ferland 2006). These line ratios are computed for a grid of density and temperature. Figure 9 shows the [Fe VII] 3760/[Fe VII] 6087 ratio in red lines (dashed line for the averaged value between the two solid lines), [Fe VII] 5160/ [Fe VII] 6087 in black, and [O III] 4959+5007/[O III] 4636 in green. The solid blue area marks the region that is consistent with the observed coronal line ratios of $\left.\left.f_{[\mathrm{Fe}} \mathrm{VII}\right] 3760 / f_{[\mathrm{Fe}} \mathrm{VII}\right] 6087=$ $0.67_{-0.13}^{+0.15}, f_{[\mathrm{Fe} \mathrm{VII}] 5160} / f_{[\mathrm{Fe} \text { VII }] 6087}<0.22\left(f_{[\mathrm{Fe} \text { VII }] 5160}(3 \sigma)<0.8 \times\right.$ $\left.10^{-16} \mathrm{erg} \mathrm{s}^{-1} \mathrm{~cm}^{-2}\right)$, and $f_{[\mathrm{O} \text { III }] 4959+5007} / f_{[\mathrm{O} \text { III }] 4636}=2.5_{-2.3}^{+2.7}$. The $n_{e}$ and $T_{e}$ values are constraint by the intersection between the green line and the solid blue area, with $n_{e} \sim(1.0-5) \times 10^{6} \mathrm{~cm}^{-3}$ and $T_{e} \sim(1.5-3) \times 10^{4} \mathrm{~K}$. The derived $T_{e}$ values suggest that the narrow line emitting gas is photoionized by the UV/optical flare.

It is noteworthy that these density and temperature values are consistent with what have been derived for coronal line AGNs (Rose et al. 2015), as well as post-shocked circumstellar medium (CSM) in type IIn SN (e.g., SN 2010jl and SN 2006jd; Stritzinger et al. 2012; Fransson et al. 2014). We discuss this ambiguity further in the following section. 


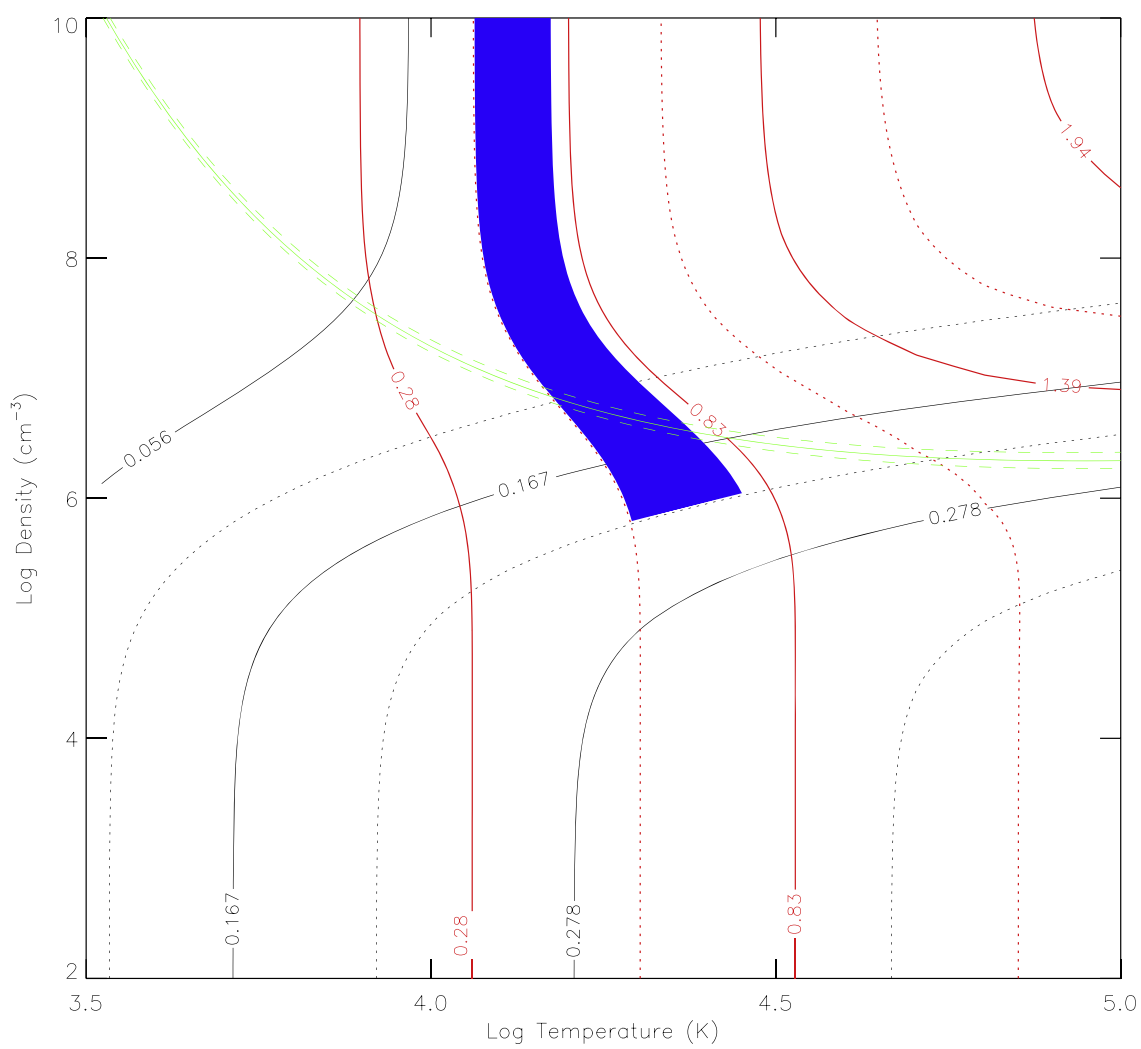

Figure 9. Computed density and temperature of the model ISM with the constraints from the forbidden line ratios.

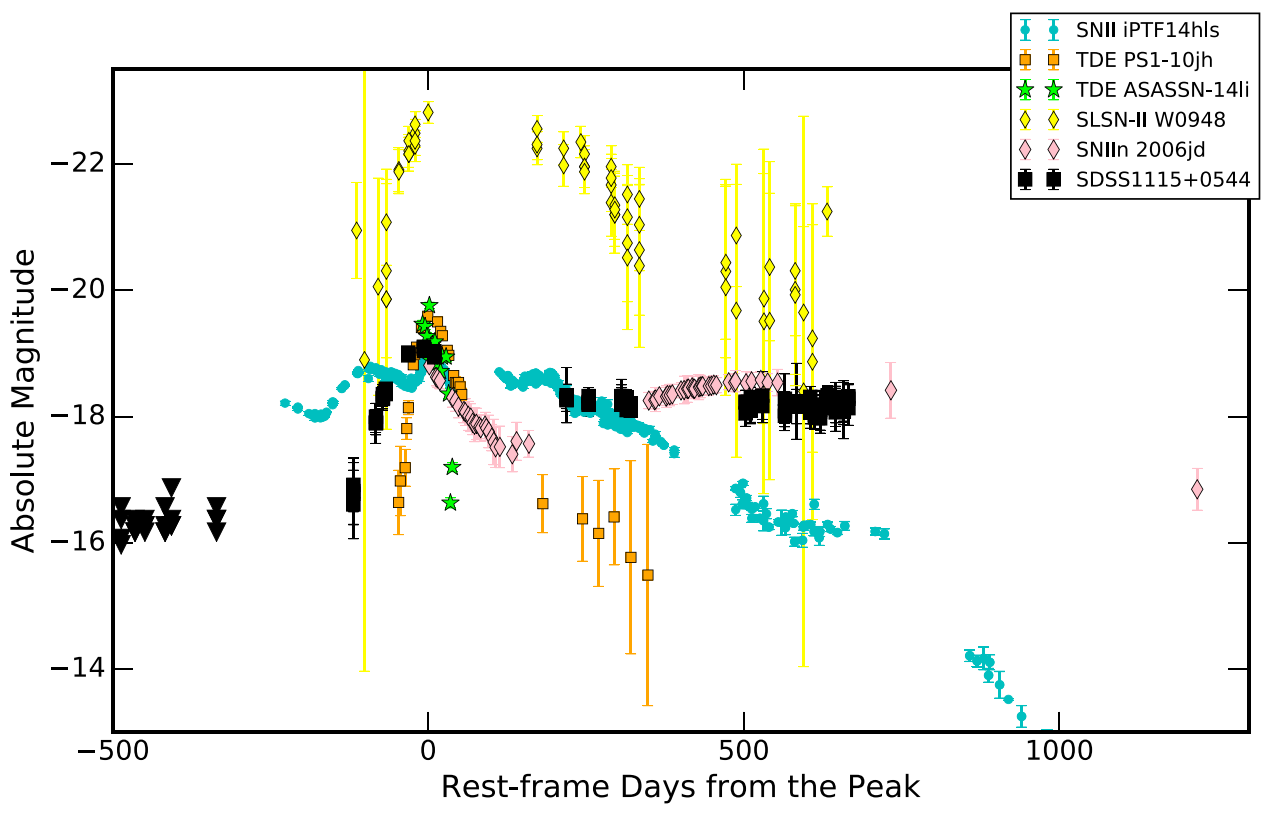

Figure 10. Comparison of the SDSS1115+0544 optical LCs with that of SNe and TDEs. The supernovae are iPTF14hls (Arcavi et al. 2017), SNIIn SN2006jd (Stritzinger et al. 2012), and SLSN-II W0948+0318 (Assef et al. 2018). The two archetypal UV/optical discovered TDEs are ASASSN-14li (Brown et al. 2017) and PS1-10jh (Gezari et al. 2012). The LCs for iPTF14hls, SN2006jd, and PS1-10jh are $r$-band, and SDSS1115+0544, ASASSN-14li, and W0948+0318are in $V$-band.

\subsection{The Flare SED from UV to Mid-IR and Its Energetics}

The observed $V$-band peak luminosity is $-19.1 \mathrm{mag}$ $\left(\nu L_{\nu}(V)=1.3 \times 10^{43} \mathrm{erg} \mathrm{s}^{-1}\right)$. With $A_{V}=1.8$, the extinction corrected optical peak luminosity is -20.9 mag $(\mathrm{AB})$. This value is comparable to that of most luminous SN IIn and TDEs (see Figure 10). The host-subtracted, peak mid-IR luminosity is $\sim-22 \mathrm{mag}(\mathrm{AB})$ at 3.4 and $4.6 \mu \mathrm{m}$ (Figure 5), very energetic and consistent with having large dust extinction. This is much higher than the peak infrared luminosities of many normal, nearby supernovae $(-12$ to $-19, \mathrm{AB})$, compiled by the SPitzer InfraRed Intensive Transients Survey (Fox et al. 2016; Tinyanont et al. 2016; Kasliwal et al. 2017). However, two mid-IR luminous supernovae indeed exist. They are SDWFSMT-1, a self-obscured supernova at $z=0.19$ with $M_{[4.5]} \sim$ -24.2 (Kozłowski et al. 2010), and SN2003ma, an unusual 


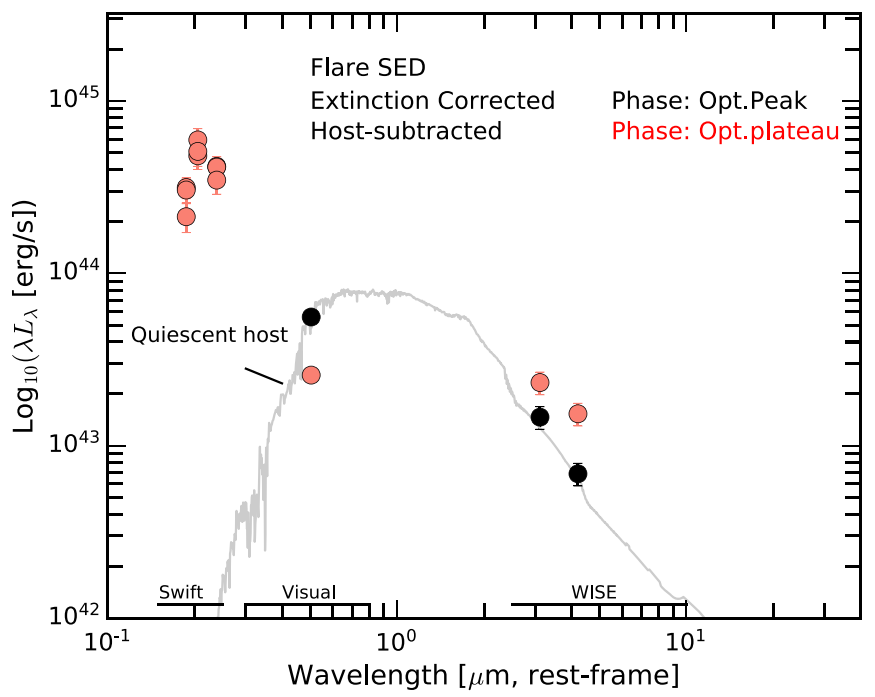

Figure 11. Full spectral energy distribution (SED) of the flare from UV to infrared wavelength. We apply the dust extinction corrections to the observed fluxes. The pink and black colors indicate the mid-IR peak and optical peak phases. All photometry are host subtracted. The Swift UV photometry is for the flare only because its host has no pre-flare UV emission.

SN IIn in a dense CSM (Rest et al. 2011). Thus, the optical and mid-IR peak luminosities alone cannot discriminate the three possible physical models among the SN IIn, TDE, and "turnon" AGN.

The Swift UV data were taken after the optical peak date of UT 2015 May $28($ MJD = 57071) and at UT 2017 July 2, 2017 July 13, and 2017 December 25. The UV fluxes do not show significant time variation (see Table 1). The extinction corrected flare SED is shown in Figure 11 as $\lambda L_{\lambda}$ from UV, optical, to mid-IR for two different phases. The pink symbols correspond to the mid-IR peak phase, while the optical LC is at the plateau (Figure 5) and the black symbols are for the earlier, optical peak phase, when the mid-IR LCs are just starting to rise.

The striking feature of the flare SED is its steep rising SED out to the UV wavelength. Clearly the bulk of the intrinsic energy from the flare event is coming out at the UV. Although the observed UV luminosity integrated from $1928 \AA$ (UVW2) to $2600 \AA$ (UVW1) is only $1.2 \times 10^{42} \mathrm{erg} \mathrm{s}^{-1}$, the extinction corrected $L_{\mathrm{UV}}$ is high, $1 \times 10^{44} \mathrm{erg} \mathrm{s}^{-1}$. Integrated over the flare SED, we infer the extinction corrected, pseudo-bolometric luminosity from the UV to infrared is $\sim 4 \times 10^{44} \mathrm{erg} \mathrm{s}^{-1}$, comparable to that of most luminous SN IIn and TDEs.

\section{Discussion: How to Distinguish an SN IIn, a TDE, and a "Turn-on" AGN?}

There are three possible physical explanations for the SDSS1115+0544 flare: an unusual type II SN, a TDE, or a "turn-on" AGN from a quiescent state. Identification of the true nature of such a flare can be ambiguous if the available observations are not sufficient. Here we make extensive comparative studies and highlight the observed properties that have and do not have discriminative powers.

\subsection{LC Comparisons with TDE and Type II SN}

Optical LCs of most normal SNe have rise timescales (from explosion to peak) of roughly $\sim 10-100$ days, with superluminous supernovae being the slowest (Yan et al. 2015, 2017;
De Cia et al. 2018). Normal SNe fade away over timescales of 100-300 days. SDSS1115+0544 clearly evolves on much longer timescales, with a bright and 600 days plateau. SN II P have post-peak plateaus, but these only last 100 days or less and then fade rapidly (Rubin et al. 2016). SDSS1115+0544 is clearly not a normal type II SN.

However, there are unusual type II (or IIn) SNe; these evolve slowly, and their ejecta interact with dense CSM. Some examples are iPTF14hls, a type II core-collapse SN (Arcavi et al. 2017); SN 2006jd, an extremely well studied SN IIn (Stritzinger et al. 2012); and WISEA J094806.56+031801.7 (W0948+0318), a possible H-rich SLSN-II in a mid-IR selected AGN (Assef et al. 2018). Figure 10 compares their LCs with those of SDSS1115+0544 over a time span of 1500 days. Of particular note, the post-peak LC of SN 2006jd (pink diamond) has the most striking similarity to that of SDSS1115+0544 (black square). Comparisons with the LCs of TDEs are also displayed in Figure 10, including two archetypal UV/optical discovered TDEs, ASASSN-14li and PS1-10jh (Brown et al. 2017; Gezari et al. 2012).

From a pure energetics point of view, the peak luminosity and total radiated energy from the SDSS1115+0544 flare have little discriminative power among the three possible physical models, and are all consistent with what have been previously observed. The strongest argument against SDSS1115+0544 being a TDE is its long timescales, for both LC and spectral line evolution. Slow rise times of 120 days and LC plateaus lasting over 600 days have not been seen among any confirmed TDEs so far. However, some TDE LCs seem to show flattening, but only in UV bands-for example, AT2018zr (PS16kh) and ASASSN-14li (Brown et al. 2017; Holoien et al. 2018; van Velzen et al. 2019). These timescales are a factor of 5 or 10 shorter. The constant $\mathrm{H} \alpha$ emission over 1 year is also unlike any known TDEs. Larger and more complete TDE samples from future transient surveys, such as ZTF, may find slow-evolving TDEs. We conclude that the SDSS1115+0544 is probably not a typical TDE, and its slow evolving LC by itself cannot rule out the possibility of being an unusual type II SN.

In the context of SN IIn versus TDE, we note several relevant points. One is that the empirical characteristics of TDEs are not yet completely known. For example, not well studied flare events include partial destruction of a star or stripping of stars (Campana et al. 2015), and events occurred in AGN host galaxies where larger accretion disks already exist prior to the disruption events (Blanchard et al. 2017; Ivanov et al. 2018). On the nuclear supernovae, they do explode near the centers of galaxies. Villarroel et al. (2017) examined the large sample of SNe discovered by PTF since 2009 and found 16 core-collapse supernovae within the projected distances $<10 \mathrm{kpc}$ from the centers of the host type-2 AGNs at $z<0.2$. Their results suggest that we cannot rule out SDSS1115+0544 being a supernova based on the physical location.

\subsection{Broad Balmer and He I Lines}

Broad Balmer emission lines with velocity widths of $3000-4000 \mathrm{~km} \mathrm{~s}^{-1}$ have been observed among type II SNe, AGNs, and TDEs. Broad He I $5876 \AA$ in the SDSS1115+0544 spectrum at +944 days was also seen in type IIn SN 2010j1 at +461 days, and also in SN 2006jd at +1544 days (Stritzinger et al. 2012; Fransson et al. 2014), as well as in TDE iPTF16fnl (Blagorodnova et al. 2017b). Therefore, these lines and their 
velocity widths cannot discriminate the possible three scenarios. However, the absence of variable narrow $\mathrm{H} \alpha$ line is evidence against the type IIn SN hypothesis because it is the hallmark of the slow moving, dense CSM. However, like the LC morphology, this does not rule out that SDSS1115+0544 may be an unusual type II SN, like iPTF14hls and SN2003ma, where the narrow $\mathrm{H} \alpha$ line was absent or emerged only at $>$ +1000 days post-peak (Rest et al. 2011; Sollerman et al. 2019).

\subsection{Coronal Fe Lines}

The formation of coronal [Fe VII] lines requires energetic ionizing photons with $E>100 \mathrm{eV}$ and a sufficient amount of gas phase $\mathrm{Fe}$ atoms that are not locked up by dust grains. ${ }^{12}$ Independent of flare models, the detections of [Fe VII] lines suggest that SDSS1115+0544 must have had a soft X-ray outburst around the time of the $V$-band flare, and the coronal line emitting regions must be relatively dust free during the period between the onset of the flare in 2015 and the last optical spectrum in 2018 May. The dust sublimation radius, the size computed from the mid-IR LC time lag, and the NLR size are consistent, $\sim 5 \times 10^{17} \mathrm{~cm}$. This supports the story that coronal lines are from the inner, ionized gas.

The potential association between coronal lines and TDEs has been extensively studied by Komossa et al. (2008, 2009), Wang et al. (2011, 2012), and Yang et al. (2013). These studies found several so-called Extreme Coronal Line Emitters (ECLE), which are believed to be possible TDE candidates. Unusually strong [Fe X], [Fe XI], [Fe XIV], and [Fe VII] emission lines are found in their initial SDSS spectra, but disappeared in the follow-up spectra taken 4-9 yr later. Some of these ECLEs also have variable broad $\mathrm{H}$ and He II emission. The timescales of optical LC and spectral evolution for these ECLEs are not constraint because of poor cadence in their spectral observations. In SDSS1115+0544, only [Fe VII] 5721, $6087 \AA$ were observed. [Fe X] would have been detected if it had a $[\mathrm{Fe} \mathrm{X}] /[\mathrm{Fe} \mathrm{VII}]$ ratio $\geqslant 1$ (Wang et al. 2012).

Coronal lines have been observed for decades among type I and II AGNs (Appenzeller \& Oestreicher 1988; Korista \& Ferland 1989; Appenzeller \& Wagner 1991; Ferguson et al. 1997; Nagao et al. 2000; Tran et al. 2000; Gelbord et al. 2009; Landt et al. 2015; Oknyansky et al. 2019). The inner wall of the AGN dust torus is usually heavily irradiated and just lies at the sublimation radius of the most refractive dust (Krolik \& Kriss 1995). From certain viewing angles, this photoionized gas can produce spectra with many coronal lines (Rose et al. 2015; Glidden et al. 2016). The [Fe VII] line luminosity from SDSS1115+0544 is comparable to that of Seyfert galaxies (Nagao et al. 2000; Gelbord et al. 2009), but its [Fe VII]/[O III] ratio of 0.4 is much higher than AGNs. This is similar to ECLEs, with unusually strong coronal lines relative to [O III] $5007 \AA$ A doublet.

Finally, coronal lines have also been detected in type IIn $\mathrm{SNe}$, especially ones with dense CSM and whose progenitor stars have experienced significant mass losses. Figure 12 compares the SDSS1115+0544 spectrum at +992 days with the late-time spectrum of SN IIn 2006jd (Stritzinger et al. 2012). Coronal Fe lines for SN2006jd only appear at several 100 days post-peak and become steadily the stronger (higher equivalent width) with time, particularly at +1544 days. This is

\footnotetext{
12 In most galactic ISM, $99 \%$ of the Fe element is in solid phase due to dust depletion (Savage \& Sembach 1996).
}

mostly due to the declining of the continuum. Similar to AGNs, photoionization by $\mathrm{X}$-rays from shocks is responsible for producing coronal emitting gas.

In conclusion, we find that high ionization coronal lines have no discriminative power among SN IIn, TDE, and AGNs.

\subsection{Evidence for "Turn-on" AGN: Constant UV Emission and Lack of $\mathrm{O}$ and $\mathrm{Ca}$ Broad Lines at Late Times}

The strongest evidence for a "turn-on" AGN model and against a type IIn SN or a TDE model is the strong, constant UV continuum emission at very late times. The host galaxy has no pre-flare UV emission, and the newly formed UV emission is associated with the flare event. Figure 13 shows the zoom-in portion of the intrinsic (dust extinction corrected) UV SED for the flare at the post-peak +700 to +992 days. The three Swift UV fluxes show little changes with time, and the UV spectral slope is extremely steep, with $f_{\lambda} \propto \lambda^{-4}$. UV emissions from supernova and TDE can be strong at early times and usually fade quickly with time. This observation supports the AGN scenario because the newly "turn-on" accretion disk can be an efficient source of UV emission.

One additional argument against SN IIn model is a lack of broad emission lines from $\mathrm{O}$ and $\mathrm{Ca}$ elements in the +992 days Keck spectrum (Figure 12). Late-time variable $\mathrm{O}$ and $\mathrm{Ca}$ line emission are very common among SN IIn. The examples of slow evolving, unusual type II SNe, such as SN 2006jd, iPTF14hls, SN2010j1, SN2003ma, and SN2005ip, all display changes in the continua and spectral features, with broad emission lines from $\mathrm{H}, \mathrm{O}$, and $\mathrm{Ca}$ elements emerging at late phases.

In summary, the flare in SDSS1115+0544 is likely a "turnon" AGN, changing from a quiescent galaxy to a type-1 AGN.

\subsection{SDSS1115+0544 as a "Turn-on" AGN: Accretion Rate Change and Space Density}

In this scenario, how much increase would be the black hole accretion rate? Taking into account the dust extinction corrections to both optical and Swift UV fluxes, the bolometric luminosity for the flare $L_{\mathrm{bol}}$ (flare) is $\sim 10^{44} \mathrm{erg} \mathrm{s}^{-1}$. If this flare is due to matter being accreted onto the black hole, we have $L_{b o l}($ flare $)=\eta \Delta \dot{m} c^{2}$ and the matter to radiative efficiency $\eta \sim 0.1$. This gives the change in accretion rate $\Delta \dot{m}$ $\sim 0.017 M_{\odot} \mathrm{yr}^{-1}$ and is a reasonable value for AGNs.

In the standard picture of BLRs around a black hole, $\mathrm{H} \beta$ line width is correlated with the central black hole mass. We adopt the following relation established by Vestergaard \& Peterson (2006), where $\log _{10} M_{\mathrm{BH}}(\mathrm{H} \beta)=\log _{10}\left\{\left[\frac{\mathrm{FWHM}(\mathrm{H} \beta)}{1000 \mathrm{~km} \mathrm{~s}^{-1}}\right]^{2}\left[\frac{L(\mathrm{H} \beta)}{10^{42} \mathrm{ergs} \mathrm{s}^{-1}}\right]^{0.63}\right\}+$ $(6.67 \pm 0.03)$. With FWHM $(\mathrm{H} \beta)=3271 \mathrm{~km} \mathrm{~s}^{-1}$ and the extinction corrected $L(\mathrm{H} \beta)=3.5 \times 10^{41} \mathrm{erg} \mathrm{s}^{-1}\left(\mathrm{~A}_{\mathrm{H} \beta}=2.1 \mathrm{mag}\right)$, the inferred $M_{\mathrm{BH}}$ is $2.6 \times 10^{7} M_{\odot}$, confirming the value based on the SDSS velocity dispersion. Using $M_{\mathrm{BH}}$ and $L_{\mathrm{bol}}$ values, we estimate the Eddington ratio $\lambda_{\mathrm{Edd}}=L_{\mathrm{bol}} / L_{\mathrm{Edd}}=0.03$, indicating low accretion rate.

If SDSS1115+0544 is a "turn-on" AGN, how are its $\mathrm{H} \alpha$ and [O III] line luminosities compared with that of known AGNs and QSOs? For low $z \sim 0.1$ type- 1 AGN, its typical $L_{5100 \AA}$ to $L_{\mathrm{H} \alpha}$ ratio is $\sim 19$ at $L_{5100 \AA} \sim 10^{44} \mathrm{erg} \mathrm{s}^{-1}$, based on the correlation derived by Greene \& Ho (2005), $L_{\mathrm{H} \alpha}=$ $(5.25 \pm 0.02) \times 10^{42}\left(\frac{L_{5100}}{10^{44}}\right)^{1.157} \mathrm{erg} \mathrm{s}^{-1}$, and the typical $L_{5100 \AA}$ to $L_{[\mathrm{O} \text { III] }}$ ratio is 320 (Kauffmann \& Heckman 2005). At the 


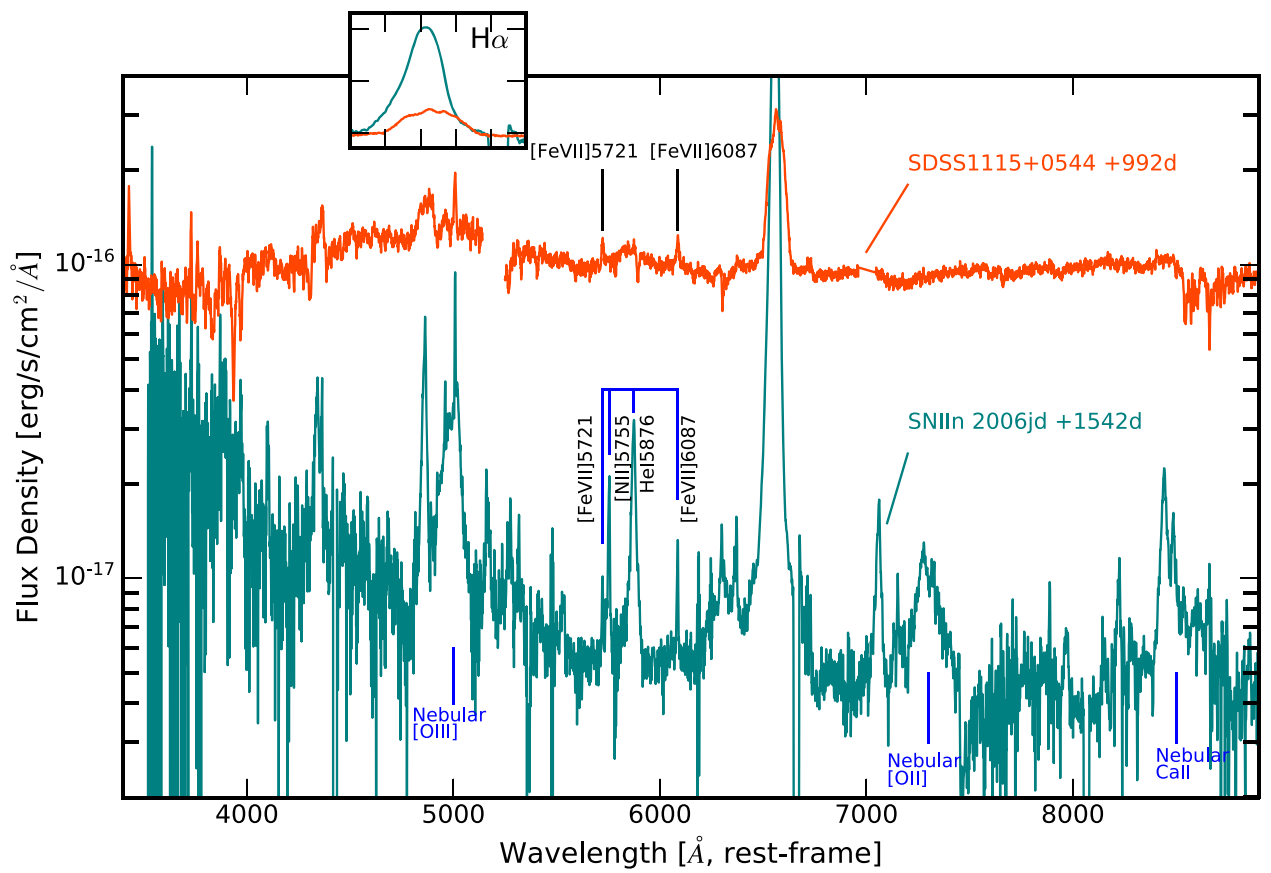

Figure 12. Comparison between the SDSS1115+0544 spectrum at +992 days post-peak and the spectrum at +1542 days for SNIIn SN2006jd (Stritzinger et al. 2012).

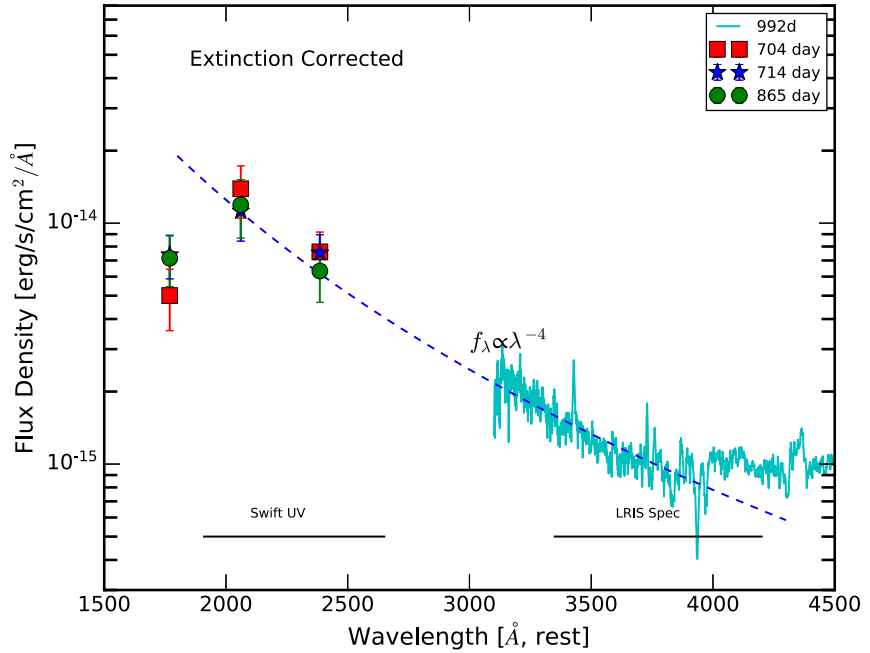

Figure 13. Dust extinction corrected UV SED of the flare measured by Swift photometry at three epochs (700-865 days from the peak) and Keck LRIS blue side of the spectrum at 992 days from the peak. The Swift UV fluxes do not show variation between epochs. Assuming no temporal variation, the spectral slope between 1900 and $4300 \AA$ is shown in a blue dashed line, with $f_{\lambda} \propto \lambda^{-4}$.

plateau phase, SDSS1115+0544 flare has a $V$-band luminosity (host subtracted) of $\sim 1 \times 10^{43} \mathrm{erg} \mathrm{s}^{-1}, L_{5100 \AA} / L_{\mathrm{H} \alpha}$ of $\sim 23$ and $L_{5100 \AA} / L_{[\mathrm{O} \mathrm{III]}} \sim 588$. This implies that SDSS1115+0544 flare has a typical $L_{\mathrm{H} \alpha}$ as that of type-1 AGN, but its $L_{[\mathrm{O} \text { III] }}$ is too weak, $1.7 \times 10^{40} \mathrm{erg} \mathrm{s}^{-1}$, just below the $10^{7} L_{\odot}$ dividing line between weak and strong AGNs, as defined by SDSS AGN studies (Kauffmann \& Heckman 2005). Thus we conclude that similar to iPTF16bco, the NLR for SDSS1115+0544 is still developing, consistent with brightening of [O III] strength. Future optical spectra will further confirm this result.

In addition, SDSS1115+0544 optical LC suggests that its continuum has changed from quiescent to the peak of flaring in only 200 days. For a radiation-dominated, Shakura-Sunyaev disk, the viscous radial inflow timescale (Shakura \& Sunyaev 1973; LaMassa et al. 2015),

$$
t_{\text {infl }}=978 y r\left[\frac{\alpha}{0.1}\right]^{-1}\left[\frac{\lambda_{\mathrm{Edd}}}{0.03}\right]^{-2}\left[\frac{\eta}{0.1}\right]^{2}\left[\frac{r}{50 r_{g}}\right]^{7 / 2}\left[\frac{M_{\mathrm{BH}, 7}}{2.0}\right],
$$

where $\alpha$ is the Shakura \& Sunyaev viscosity parameter and $r_{g}$ is the gravitational radius $\left(G M / c^{2}\right)$. If $\alpha, \eta$, and $r$ are assumed to be $0.1,0.1$, and $50 r_{g}$ (optical emission typically comes from 50 to $100 r_{g}$ ), respectively, the viscous inflow timescale at $50 r_{g}$ is 978 years, much longer than the 200 day continuum "turnon" timescale measured from the SDSS1115+0544 LC. This "viscosity crisis" has been noted and is the focus of a recent paper by Lawrence (2018).

SDSS1115+0544 is a case of a quiescent LINER transitioning into an AGN in a short timescale $<1 \mathrm{yr}$. It is the first rapid "turn-on" AGN with a well constraint rise timescale based on its extensive CRTS LC. iPTF16bco is another such an event at $z=0.237$ (Gezari et al. 2017). Its quiescent host is also a LINER, and the transformation was characterized by optical flare (iPTF LC), increasing UV continuum emission and newly formed broad $\mathrm{H} \alpha$ emission, similar to that of SDSS1115+0544. It attempts to speculate that the short "turn-on" timescales found in these two cases may be associated with LINERs where rapid accretion rate changes or disk instability may likely occur. However, better understanding requires more such events to be discovered by ongoing and future transient surveys.

AGNs have been known and studied for more than half a century. The actively accreting phase (i.e., AGN phase) is thought to last roughly a few times $10^{7}$ yr (so-called duty cycle; Combes 2000; Haehnelt \& Rees 1993). "Turn-on" AGNs like SDSS1115+0544 and iPTF16bco have a flare timescale of 200-300 days. By scaling these two timescales, we infer that "turn-on" AGN is only $10^{-7}$ of the parent AGN population. 
Counting low luminosity AGNs, the space density of Seyfert 1 is roughly $10^{-4} \mathrm{Mpc}^{-3}$ at low redshift (Osterbrock \& Ferland 2006). Thus, events like SDSS1115+0544 should have space density of $10^{-11} \mathrm{Mpc}^{-3}$. However, without any systematic searches, two rapid "turn-on" type-1 AGN/QSO have been found with the flaring peak time separated by only a year. This may indicate that our calculation could significantly underestimate the true value. Perhaps the AGN duty cycle is not the proper timescale to use for this calculation, because a duty cycle of $10^{7} \mathrm{yr}$ only counts for the total time when AGN is active. This does not distinguish various flare episodes that will have a distribution of different timescales. Upcoming large transient surveys should produce actual measurements of this distribution.

\section{Summary}

SDSS1115+0544 is a transient first selected as a part of our systematic search for infrared flare galaxies within the SDSS area. The host galaxy is a quiescent, early-type galaxy at $z=$ 0.0899 with $M_{\text {stars }} \sim 3.5 \times 10^{10} M_{\odot}, M_{\mathrm{BH}} \sim 2 \times 10^{7} M_{\odot}$, $\mathrm{SFR}_{24 \mu \mathrm{m}}$ of $\sim 0.2 M_{\odot} \mathrm{yr}^{-1}$. Its SDSS optical spectrum has the characteristics of a LINER with a non-accreting black hole and weak narrow forbidden emission lines. In January 2015, SDSS1115+0544 underwent a strong optical flare, with a rise of 2.5 mag ( $V$-band) over $\sim 120$ days. Declining from the peak magnitude (host subtracted) after +200 days post-peak, its optical LC reached a prolonged plateau, $1.8 \mathrm{mag}$ brighter than the quiescent level and lasting over 600 days. This optical flare was followed by mid-infrared flares at 3.4 and $4.6 \mu \mathrm{m}$ with amplitudes of $\geqslant 0.5$ mag over 600 days. The time lag between the infrared and optical peak is $\sim 180$ days. SDSS $1115+0544$ displayed excess UV continuum emissions that were measured by Swift UV observations at three epochs, $>+730$ days post-peak (optical LC). This excess UV emission shows no temporal variation, likely to be on a plateau similar to the optical. A series of optical spectra of this flare event were obtained between 2016 January 6 and 2018 May 13. Compared to the SDSS spectrum taken in 2011, the new spectra taken after the optical flare show a rich suite of newly formed emission lines, including broad Balmer and helium lines with velocity widths $\sim 3750 \mathrm{~km} \mathrm{~s}^{-1}$, and narrow high ionization forbidden lines such as [Fe VII], [Ne V].

We made extensive comparisons of the SDSS1115+0544 properties with known TDEs and unusual type II SN. Although the newly formed coronal Fe lines link this event to ECLE, the slow evolving optical LC over 1200 days and its non-variant broad Balmer lines at very late times argue against the SDSS1115+0544 flare being a typical UV/optically selected TDE. We find that LC energetics, morphology, timescale, as well as spectral features, including broad Balmer emission and narrow coronal lines, cannot serve as clear discriminants between "turn-on" AGN and an unusual type II SN. This highlights the ambiguity in identifications of many transients discovered in the nuclear regions of quiescent galaxies.

In the case of SDSS1115+0544, the strongest evidence against the scenario of unusual type II SNe is the strong UV continuum observed at +700 to +900 days without any temporal variations. In addition, our Keck spectrum at +992 days does not show any broad $\mathrm{O}$ and $\mathrm{Ca}$ emission lines commonly seen in the late-time spectra of type II SN, as in examples of unusual type II SN SN2006jd, iPTF14hls, SN2005ip, and SN2010jl. We conclude that the flare in SDSS1115+0544 is from a new "turn-on" accretion disk around the supermassive black hole, transforming the previously quiescent early-type galaxy into a type-1 AGN, with a sub-Eddington accretion rate of $\sim 0.02 M_{\odot} \mathrm{yr}^{-1}$.

This new "turn-on" accretion disk produces significantly soft $\mathrm{X}$-ray and UV photons that are responsible for continuum flares, the emergence of broad Balmer emission lines, as well as narrow high ionization metal lines. The radiative feedback from the accretion can drive gas outflows, naturally producing a broad Balmer line emitting region with a size likely $<10^{16} \mathrm{~cm}$. SDSS $1115+0544$ contains a substantial dusty ISM, with a column density of $3 \times 10^{21} \mathrm{~cm}^{-2}\left(A_{V}=1.8\right)$. This dusty medium produces the infrared echoes of the optical flare, generating the infrared variability. The time lag between the IR and optical LCs implies that the dusty ISM is about $5 \times 10^{17} \mathrm{~cm}$ from the central accretion disk.

It is interesting to note that if the rise timescale determines the AGN "turn-on" timescale, it is very short for SDSS1115+0544, $<200$ days from the well-sampled CRTS LC. This discovery suggests that the changing state phenomenon among AGNs could be associated with violent instabilities in accretion disks occurring on timescales of a few 100 days. More events such as SDSS1115 +0544 from future transient surveys should provide better constraints on the event rates as well as theoretical understanding of accretion disks around supermassive black holes.

We thank A. Ho, M. Kuhn from Caltech, T. Hung, and S. Frederick from University of Maryland for helping with taking some of the spectral observations. This research has made use of the NASA/IPAC Infrared Science Archive, which is operated by the Jet Propulsion Laboratory, California Institute of Technology, under contract with the National Aeronautics and Space Administration. We also use the data products from the Wide-field Infrared Survey Explorer, which is a joint project of the University of California, Los Angeles, and the Jet Propulsion Laboratory/California Institute of Technology, funded by the National Aeronautics and Space Administration. This paper also utilized the publicly available SDSS data sets. Funding for the SDSS and SDSS-II has been provided by the Alfred P. Sloan Foundation, the Participating Institutions, the National Science Foundation, the US Department of Energy, the National Aeronautics and Space Administration, the Japanese Monbukagakusho, the Max Planck Society, and the Higher Education Funding Council for England. The SDSS website is http://www.sdss.org/. The SDSS is managed by the Astrophysical Research Consortium for the Participating Institutions. The participating institutions are the American Museum of Natural History, Astrophysical Institute Potsdam, the University of Basel, the University of Cambridge, Case Western Reserve University, the University of Chicago, Drexel University, Fermilab, the Institute for Advanced Study, the Japan Participation Group, Johns Hopkins University, the Joint Institute for Nuclear Astrophysics, the Kavli Institute for Particle Astrophysics and Cosmology, the Korean Scientist Group, the Chinese Academy of Sciences (LAMOST), Los Alamos National Laboratory, the Max-Planck-Institute for Astronomy (MPIA), the Max-Planck-Institute for Astrophysics (MPA), New Mexico State University, Ohio State University, the University of Pittsburgh, the University of Portsmouth, Princeton University, the United States Naval Observatory, and the University of Washington. This research has made use of the NASA/IPAC Extragalactic Database (NED), which is operated by the Jet Propulsion Laboratory, California Institute of Technology, under contract with the National Aeronautics 
and Space Administration. Some of the data presented herein were obtained at the W. M. Keck Observatory, which is operated as a scientific partnership among the California Institute of Technology, the University of California, and the National Aeronautics and Space Administration. The observatory was made possible by the generous financial support of the W. M. Keck Foundation. The authors wish to recognize and acknowledge the very significant cultural role and reverence that the summit of Maunakea has always had within the indigenous Hawaiian community. We are most fortunate to have the opportunity to conduct observations from this mountain.

Facilities: Palomar, Keck, WISE, Swift.

\section{ORCID iDs}

Lin Yan (ib https://orcid.org/0000-0003-1710-9339

Tinggui Wang (i) https://orcid.org/0000-0002-1517-6792

Daniel Stern (ib) https://orcid.org/0000-0003-2686-9241

Liming Dou (iD https://orcid.org/0000-0002-4757-8622

M. M. Kasliwal (i) https://orcid.org/0000-0002-5619-4938

\section{References}

Andrillat, Y. 1968, AJ, 73, 862

Appenzeller, I., \& Oestreicher, R. 1988, AJ, 95, 45

Appenzeller, I., \& Wagner, S. J. 1991, A\&A, 250, 57

Arcavi, I., Gal-Yam, A., Sullivan, M., et al. 2014, ApJ, 793, 38

Arcavi, I., Howell, D. A., Kasen, D., et al. 2017, Natur, 551, 210

Assef, R. J., Prieto, J. L., Stern, D., et al. 2018, ApJ, 866, 26

Barvainis, R. 1987, ApJ, 320, 537

Bellm, E. C., Kulkarni, S. R., Graham, M. J., et al. 2019, PASP, 131, 018002

Blagorodnova, N., Gezari, S., Hung, T., et al. 2017a, ApJ, 844, 46

Blagorodnova, N., Gezari, S., Hung, T., et al. 2017b, ApJ, 844, 46

Blanchard, P. K., Nicholl, M., Berger, E., et al. 2017, ApJ, 843, 106

Bon, E., Zucker, S., Netzer, H., et al. 2016, ApJS, 225, 29

Brown, J. S., Holoien, T. W.-S., Auchettl, K., et al. 2017, MNRAS, 466, 4904

Calzetti, D. 2013, in Star Formation Rate Indicators, ed. J. Falcón-Barroso \&

J. H. Knapen (Cambridge: Cambridge Univ. Press), 419

Campana, S., Mainetti, D., Colpi, M., et al. 2015, A\&A, 581, A17

Caplan, J., \& Deharveng, L. 1986, A\&A, 155, 297

Cid Fernandes, R., Stasińska, G., Schlickmann, M. S., et al. 2010, MNRAS, 403, 1036

Combes, F. 2000, in ASP Conf. Ser.197, Dynamics of Galaxies: from the Early Universe to the Present, ed. F. Combes, G. A. Mamon, \& V. Charmandaris (San Francisco, CA: ASP), 15

Comparat, J., Maraston, C., Goddard, D., et al. 2017, arXiv:1711.06575

De Cia, A., Gal-Yam, A., Rubin, A., et al. 2018, ApJ, 860, 100

Dong, X., Wang, T., Wang, J., et al. 2008, MNRAS, 383, 581

Dou, L., Wang, T., Yan, L., et al. 2017, ApJL, 841, L8

Drake, A. J., Djorgovski, S. G., Mahabal, A., et al. 2009, ApJ, 696, 870

Ferguson, J. W., Korista, K. T., Baldwin, J. A., \& Ferland, G. J. 1997, ApJ, 487, 122

Fox, O. D., Johansson, J., Kasliwal, M., et al. 2016, ApJL, 816, L13 Fransson, C., Ergon, M., Challis, P. J., et al. 2014, ApJ, 797, 118 Fremling, C., Sollerman, J., Taddia, F., et al. 2016, A\&A, 593, A68 French, K. D., Arcavi, I., \& Zabludoff, A. 2016, ApJL, 818, L21

Gelbord, J. M., Mullaney, J. R., \& Ward, M. J. 2009, MNRAS, 397, 172

Gezari, S., Chornock, R., Rest, A., et al. 2012, Natur, 485, 217

Gezari, S., Hung, T., Cenko, S. B., et al. 2017, ApJ, 835, 144

Glidden, A., Rose, M., Elvis, M., \& McDowell, J. 2016, ApJ, 824, 34

Graham, M. J., Djorgovski, S. G., Stern, D., et al. 2015, Natur, 518, 74

Graham, M. J., Kulkarni, S. R., Bellm, E. C., et al. 2019, arXiv:1902.01945

Greene, J. E., \& Ho, L. C. 2005, ApJ, 630, 122

Haehnelt, M. G., \& Rees, M. J. 1993, MNRAS, 263, 168

Hills, J. G. 1976, ApL, 17, 95

Hogg, D. W., Baldry, I. K., Blanton, M. R., \& Eisenstein, D. J. 2002, arXiv: astro-ph/0210394

Holoien, T. W.-S., Huber, M. E., Shappee, B. J., et al. 2018, arXiv:1808.02890

Holoien, T. W.-S., Kochanek, C. S., Prieto, J. L., et al. 2016, MNRAS, 455, 2918
Holoien, T. W.-S., Prieto, J. L., Bersier, D., et al. 2014, MNRAS, 445, 3263 Ivanov, P. B., Zhuravlev, V. V., \& Papaloizou, J. C. B. 2018, MNRAS, 481,3470

Jiang, N., Dou, L., Wang, T., et al. 2016, ApJL, 828, L14

Jiang, N., Wang, T., Yan, L., et al. 2017, ApJ, 850, 63

Jun, H. D., Stern, D., Graham, M. J., et al. 2015, ApJL, 814, L12

Kaiser, N., Aussel, H., Burke, B. E., et al. 2002, Proc. SPIE, 4836, 154

Kankare, E., Kotak, R., Mattila, S., et al. 2017, NatAs, 1, 865

Kasliwal, M. M., Bally, J., Masci, F., et al. 2017, ApJ, 839, 88

Kauffmann, G., \& Heckman, T. M. 2005, RSPTA, 363, 621

Kewley, L. J., Groves, B., Kauffmann, G., \& Heckman, T. 2006, MNRAS, 372, 961

Komossa, S., Zhou, H., Rau, A., et al. 2009, ApJ, 701, 105

Komossa, S., Zhou, H., Wang, T., et al. 2008, ApJL, 678, L13

Korista, K. T., \& Ferland, G. J. 1989, ApJ, 343, 678

Kozłowski, S., Kochanek, C. S., Stern, D., et al. 2010, ApJ, 722, 1624

Krolik, J. H., \& Kriss, G. A. 1995, ApJ, 447, 512

LaMassa, S. M., Cales, S., Moran, E. C., et al. 2015, ApJ, 800, 144

Landt, H., Ward, M. J., Steenbrugge, K. C., \& Ferland, G. J. 2015, MNRAS, 454, 3688

Law, N. M., Kulkarni, S. R., Dekany, R. G., et al. 2009, PASP, 121, 1395

Lawrence, A. 2018, NatAs, 2, 102

Lyutyj, V. M., Oknyanskij, V. L., \& Chuvaev, K. K. 1984, SvAL, 10, 335

MacLeod, C. L., Ross, N. P., Lawrence, A., et al. 2016, MNRAS, 457, 389

Mainzer, A., Bauer, J., Cutri, R. M., et al. 2014, ApJ, 792, 30

Mathur, S., Denney, K. D., Gupta, A., et al. 2018, ApJ, 866, 123

Mattila, S., Pérez-Torres, M., Efstathiou, A., et al. 2018, Sci, 361, 482

McConnell, N. J., \& Ma, C.-P. 2013, ApJ, 764, 184

Nagao, T., Taniguchi, Y., \& Murayama, T. 2000, AJ, 119, 2605

Oke, J. B., Cohen, J. G., Carr, M., et al. 1995, PASP, 107, 375

Oke, J. B., \& Gunn, J. E. 1983, ApJ, 266, 713

Oknyanskij, V., \& Lyuty, V. 2007, PZP, 7, 28, http://www.astronet.ru/db/ varstars $/ \mathrm{msg} / 1224297$

Oknyanskij, V. L. 1978, PZ, 21, 71

Oknyansky, V. L., Winkler, H., Tsygankov, S. S., et al. 2019, MNRAS, 483, 558

Osterbrock, D. E., \& Ferland, G. J. 2006, Astrophysics of gaseous nebulae and active galactic nuclei (Sausalito, CA: University Science Books)

Penston, M. V., \& Perez, E. 1984, MNRAS, 211, 33P

Phinney, E. S. 1989, in IAU Symp. 136, The Center of the Galaxy, ed. M. Morris (Dordrecht: Kluwer), 543

Polletta, M., Tajer, M., Maraschi, L., et al. 2007, ApJ, 663, 81

Rau, A., Kulkarni, S. R., Law, N. M., et al. 2009, PASP, 121, 1334

Rees, M. J. 1988, Natur, 333, 523

Rest, A., Foley, R. J., Gezari, S., et al. 2011, ApJ, 729, 88

Robitaille, T. P., \& Whitney, B. A. 2010, ApJL, 710, L11

Rose, M., Elvis, M., \& Tadhunter, C. N. 2015, MNRAS, 448, 2900

Ruan, J. J., Anderson, S. F., Green, P. J., et al. 2016, ApJ, 825, 137

Rubin, A., Gal-Yam, A., De Cia, A., et al. 2016, ApJ, 820, 33

Runnoe, J. C., Cales, S., Ruan, J. J., et al. 2016, MNRAS, 455, 1691

Savage, B. D., \& Sembach, K. R. 1996, ARA\&A, 34, 279

Shakura, N. I., \& Sunyaev, R. A. 1973, A\&A, 24, 337

Singh, R., van de Ven, G., Jahnke, K., et al. 2013, A\&A, 558, A43

Sollerman, J., Taddia, F., Arcavi, I., et al. 2019, A\&A, 621, 30

Stern, D., Assef, R. J., Benford, D. J., et al. 2012, ApJ, 753, 30

Stern, D., McKernan, B., Graham, M. J., et al. 2018, ApJ, 864, 27

Stritzinger, M., Taddia, F., Fransson, C., et al. 2012, ApJ, 756, 173

Tadhunter, C., Spence, R., Rose, M., Mullaney, J., \& Crowther, P. 2017, NatAs, 1, 0061

Tinyanont, S., Kasliwal, M. M., Fox, O. D., et al. 2016, ApJ, 833, 231

Tohline, J. E., \& Osterbrock, D. E. 1976, ApJL, 210, L117

Tonry, J. L., Denneau, L., Heinze, A. N., et al. 2018, PASP, 130, 064505

Tran, H. D., Cohen, M. H., \& Villar-Martin, M. 2000, AJ, 120, 562

van Velzen, S., Farrar, G. R., Gezari, S., et al. 2011, ApJ, 741, 73

van Velzen, S., Gezari, S., Cenko, S., et al. 2019, ApJ, 872, 198

Vestergaard, M., \& Peterson, B. M. 2006, ApJ, 641, 689

Villarroel, B., Nyholm, A., Karlsson, T., et al. 2017, ApJ, 837, 110

Wang, T.-G., Zhou, H.-Y., Komossa, S., et al. 2012, ApJ, 749, 115

Wang, T.-G., Zhou, H.-Y., Wang, L.-F., Lu, H.-L., \& Xu, D. 2011, ApJ, 740,85

Wright, E. L., Eisenhardt, P. R. M., Mainzer, A. K., et al. 2010, AJ, 140, 1868

Yan, L., Donoso, E., Tsai, C.-W., et al. 2013, AJ, 145, 55

Yan, L., Lunnan, R., Perley, D. A., et al. 2017, ApJ, 848, 6

Yan, L., Quimby, R., Ofek, E., et al. 2015, ApJ, 814, 108

Yang, C.-W., Wang, T.-G., Ferland, G., et al. 2013, ApJ, 774, 46

Yang, Q., Wu, X.-B., Fan, X., et al. 2017, arXiv:1711.08122 